\title{
Surface Transport Processes in Charged Porous Media
}

\author{
Jorge Gabitto $^{1 *}$ and Costas Tsouris ${ }^{2}$ \\ ${ }^{1}$ Department of Chemical Engineering \\ Prairie View A\&M University \\ Prairie View, TX 77446 \\ jgabitto@aol.com \\ ${ }^{2}$ Oak Ridge National Laboratory \\ Oak Ridge TN 37831-6181 \\ *Author to whom correspondence should be addressed
}

Submitted for publication in

Journal of Colloid and Interface Science

February 2017

Notice: This manuscript has been authored by UT-Battelle, LLC under Contract No. DE-AC0500OR22725 with the US Department of Energy. The United States Government retains and the publisher, by accepting the article for publication, acknowledges that the United States Government retains a non-exclusive, paid-up, irrevocable, world-wide license to publish or reproduce the published form of this manuscript, or allow others to do so, for United States Government purposes. The Department of Energy will provide public access to these results of federally sponsored research in accordance with the DOE Public Access Plan (http://energy.gov/downloads/doe-public-access-plan). 


\title{
Surface Transport Processes in Charged Porous Media
}

\author{
Jorge Gabitto $^{1 *}$ and Costas Tsouris ${ }^{2}$ \\ ${ }^{1}$ Department of Chemical Engineering \\ Prairie View A\&M University \\ Prairie View, TX 77446 \\ jgabitto@aol.com
}

${ }^{2}$ Oak Ridge National Laboratory, Oak Ridge TN 37831-6181

*Author to whom correspondence should be addressed

\section{ABSTRACT}

Surface transport processes are very important in chemistry, colloidal sciences, engineering, biology, and geophysics. Natural or externally produced charges on surfaces create electrical double layers (EDLs) in the solid-liquid interface. The existence of the EDLs produces several complex processes including bulk and surface transport of ions. In this work, a model is presented to simulate bulk and transport processes in homogeneous porous media comprising big pores. It is based on a theory for capacitive charging by ideally polarizable porous electrodes without Faradaic reactions or specific adsorption of ions. A volume averaging technique is used to derive the averaged transport equations in the limit of thin electrical double layers. Description of the EDL between the electrolyte solution and the charged wall is accomplished using the Gouy-Chapman-Stern (GCS) model. The surface transport terms enter into the average equations due to the use of boundary conditions for diffuse interfaces. Two extra surface transports terms appear in the closed average equations. One is a surface diffusion term equivalent to the transport process in non-charged porous media. The second surface transport term is a migration term unique to charged porous media. The effective bulk and transport parameters for isotropic porous media are calculated solving the corresponding closure problems.

Keywords: Surface transport, Volume average, Porous media

\section{INTRODUCTION}

Surface transport phenomena in charged porous media are important in many scientific disciplines such as engineering, electrochemistry, biology, geophysics, and colloidal science (Johnson and Sen, 1988; Revil and Glover, 1997; Lyklema and Minor, 1997; Dukhin et al.; 2001; Corry et al., 2003, Bazant et al., 2004; Chu and Bazant, 2006; among many others). Even though these disciplines deal with different problems there are many basic similarities in the studied physical phenomena. The presence of an electrical double layer (EDL) generated by natural or induced charges on the solid surface is a common feature of many of these different systems.

At this point we introduce a set of definitions in order to avoid confusion because similar terms are used in different ways across different scientific disciplines. We want also to state 
precisely the EDL model used throughout this work. We consider a model presenting the following features, see Figure 1:

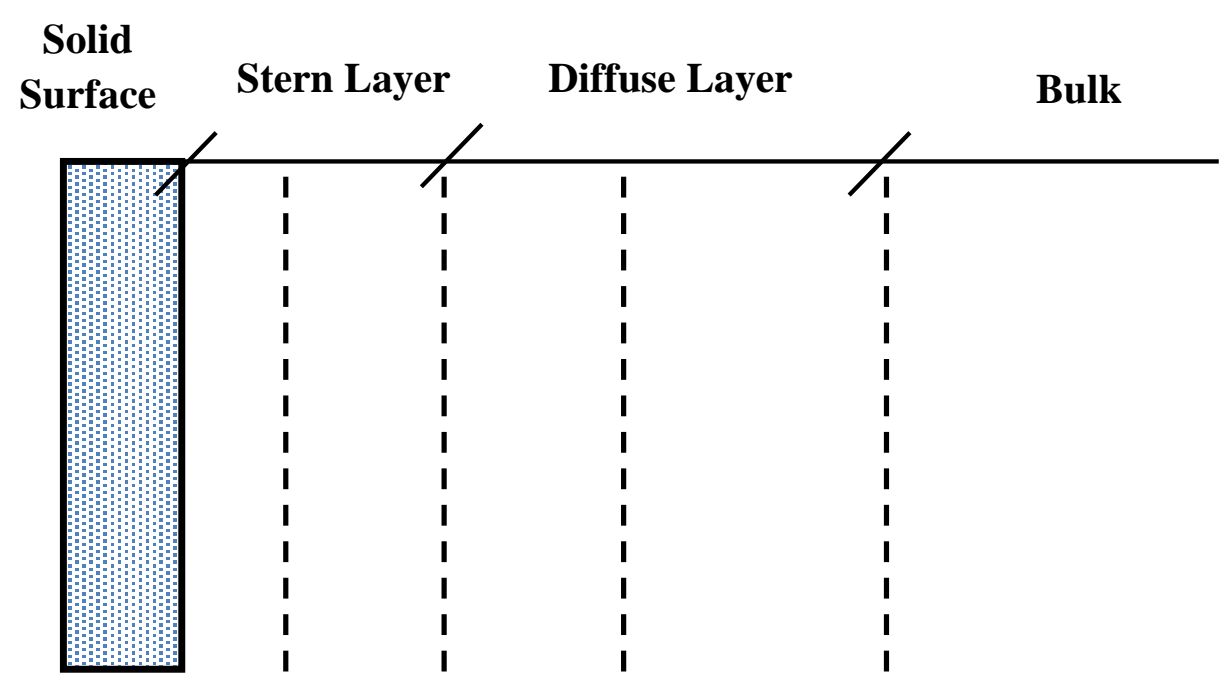

\section{IHP OHP Slip Plane}

Figure 1. EDL model schematic.

1) There is a solid hard surface,-such that there are electrical charges distributed on the solidliquid interface.

2) There may be specifically (chemically) adsorbed ions on the surface. The inner Helmholtz plane (IHP) is the locus of the radii of these chemically adsorbed ions.

3) There is a layer of counterions located, on average, a distance from the electrode equal to the hydrated radius of the counterion. We can also consider a layer of water molecules adjacent to the solid surface. In the latter case, the distance to the electrode will be the hydrated radius of the ions (a) plus the diameter of the water molecules (h). The outer Helmholtz plane (OHP) is the locus of the radii of the counterions, located either at a distance a from the electrode or at a distance $\mathrm{a}+\mathrm{h}$.

4) The slipping (or shear or slip) plane separates mobile fluid from fluid that remains attached to the surface; i.e., within the slipping plane region the fluid is hydrodynamically stagnant.

5) In this work, we will follow the Bockris et al. (2000) model; therefore, the Stern layer extends up to the OHP. The diffuse layer will be the region beyond the OHP until the region of constant properties, i.e., the bulk of the solution.

\subsection{Role of Surface Conduction in Colloidal Sciences}

Lyklema and Minor (1998) presented a review on the role of surface conduction in electrokinetics. Surface conduction was defined as the excess conduction that takes place in dispersed systems due to the presence of electrical double layers (EDLs). The existence of EDLs reflects the local presence of excess charges that may move under the influence of electric fields tangentially to the surface. Surface conduction is quantified in terms of a surface conductivity 
$\mathrm{K}_{\sigma}$, which is the surface equivalent of the bulk conductivity $\mathrm{K}_{\mathrm{L}}$. In contrast to $\mathrm{K}_{\mathrm{L}}, \mathrm{K}_{\sigma}$ is an excess quantity (Lyklema and Minor, 1998). It is important to notice that Lyklema and Minor (1998) considered the area between the OHP and the slip plane to be part of the Stern layer. Therefore, there are potentially mobile ions within the Stern layer while, according to the Bockris et al. (2000) model, the only ions inside the Stern layer are the specifically adsorbed ions. This issue can lead to confusion and errors because the electrical potential, customarily measured experimentally in electrokinetics as the $\zeta$-potential, is the potential at the slipping plane and not always at the OHP (Zukoski IV and Saville, 1985 and 1986.)

Dukhin et al. (2001) proposed a generalized standard electrokinetic model (GSEM). The suggested model assumed the occurrence of conductivity in the stagnant layer. The author considered that this surface conductivity could be produced by two different mechanisms, ion fluxes in the region between the OHP and the slipping plane and movement of specifically adsorbed ions within the region between the solid surface and the OHP. Within the framework of the GSEM, the EDL is described by at least two parameters: $\zeta$-potential and surface conductivity $\left(\mathrm{K}_{\sigma}\right)$. Dukhin et al. (2001) also proposed experiments in the vicinity of the isoelectric point for the discrimination between the two different mechanisms of ionic conductivity in the stagnant layer.

Lyklema and Minor (1998) defined the following dimensionless number:

$$
\mathrm{Du} \equiv \frac{\mathrm{K}_{\sigma}}{\mathrm{d} \mathrm{K}_{\mathrm{L}}} \text {. }
$$

The authors called it Dukhin's number in order to acknowledge Dukhin's important contributions to EDL relaxation and electrokinetic phenomena. This dimensionless number was originally introduced by Bikerman (1940) and used by other authors. Dukhin himself called it Rel. In Eq. (1), d is a characteristic length; for movement of spherical particles in an electric field, for example, $d$ is the particle diameter; for transport in a cylindrical pore, $d$ is the diameter of the pore, etc. (Bazant et al., 2004). The value of the Dukhin number has also been used as a criterion for neglecting surface transport processes. Biesheuvel and Bazant (2010) stated that, in many cases, the thin double-layer approximation also justifies the neglect of tangential surface transport through the double layers compared to bulk transport within the quasi-neutral pores, as long as the surface conductance $\mathrm{K}_{\sigma}$ remains much smaller than the bulk conductance $\mathrm{K}_{\mathrm{L}}$ (small Dukhin number).

Bazant and co-workers have analyzed the presence of surface transport (Bazant et al., 2004; Chu and Bazant, 2006; Chu and Bazant, 2007). Bazant et al. (2004) studied a model problem where a sudden voltage is applied to a symmetric binary electrolyte located between parallelplate blocking electrodes. The authors included a historical review including electrochemistry, colloidal science, and microfluidics. Concerning the influence of the Du number, Bazant et al. (2004) stated that, for $\mathrm{Du}<<1$, the EDL remains in its equilibrium state at a constant $\zeta$-potential but, for $\mathrm{Du}>>1$, it becomes distorted as surface conduction draws current lines into the EDL. Chu and Bazant (2006) analyzed the response to a uniform electric field of an uncharged, ideally polarizable metallic sphere or cylinder located in a symmetric, binary electrolyte. Their mathematical treatment was based on the Poisson-Nernst-Planck (PNP) equations of the dilute solution theory with focus on the nonlinear regime, where the applied voltage across the conductor is larger than the thermal voltage. In such strong electric fields, the classical model 
predicts that the double layer adsorbs enough ions to produce bulk concentration gradients and surface conduction. The authors also presented a general derivation of surface conservation laws in the thin double-layer limit, which provides effective boundary conditions on the solid-liquid interface. Chu and Bazant (2007) provided a mathematically rigorous derivation of surface conservation laws at diffuse interfaces based on an asymptotic analysis of transport processes in the boundary layer. The authors also derived general equations for the surface and normal fluxes that appear in surface conservation laws. Chu and Bazant (2007) used nonequilibrium thermodynamics to formulate surface conservation laws in terms of chemical potentials. The authors also derived surface conservation laws for several examples involving transport in dilute and concentrated solutions of charge and uncharged solutes.

Mirzadeh et al. (2014) used direct numerical simulations of the Poisson-Nernst-Planck equations to study the charging kinetics of porous electrodes and to evaluate the predictive capabilities of effective circuit models. The authors identified surface conduction as a mechanism which can "short circuit" the high-resistance electrolyte in the bulk of the pores, thus accelerating the charging dynamics and boosting power densities. This effect was found to hold only for electrode morphologies with continuous conducting surfaces in the charging direction.

\subsection{Role of EDLs in Geophysics}

Revil and co-workers presented several studies related to ion transport in porous media (Revil et al., 1996; Revil et al., 1997; Revil and Glover, 1997; Revil and Glover, 1998; Revil, 1999; Leroy and Revil, 2004). They studied the case of porous media consisting of an insulating matrix and an interconnected pore volume saturated with an electrolyte. In contact with electrolytes, mineral surfaces get an excess of charge that is balanced by mobile ions in the electrical diffuse layer above the solid surface.

Revil et al. (1996) presented a self-consistent set of equations to study the electrical conductivity, streaming potential, and the membrane potential as a function of the EDL structure and properties of the porous medium. The authors assumed that there is no ion movement inside the Stern layer or through the active sites on the solid; therefore, the extra conductivity occurs within the diffuse layer only. This extra conductivity within the diffuse layer is accounted for using a specific conductance parameter $\left(\Sigma_{\mathrm{S}}\right)$. The authors followed Schwartz et al. (1989) defining $\sum_{\mathrm{S}}$ as:

$$
\Sigma_{\mathrm{S}}=\int_{0}^{\chi_{D}}\left(\sigma(\chi)-\sigma_{B}\right) d \chi
$$

Here, $\sigma_{B}$ is the conductivity in the bulk of the solution, $\sigma(\chi)$ is the conductivity within the diffuse layer, $\chi_{D}$ is the size of the diffuse layer, and $\chi$ is the integration variable along the length of the diffuse layer. Revil and Glover (1997) presented a model describing ionic electrical conduction in porous media, with particular emphasis given to surface conduction. The authors stated that electrical conduction in the diffuse layer can contribute substantially to the effective electrical conductivity of the porous medium. Revil and Glover (1997) concluded that the specific surface conductance used to describe the surface electrical conduction is related to the aforementioned properties, via the electrical surface potential which is dependent upon the electrolyte concentration and $\mathrm{pH}$. The authors did not consider electrical conduction in the Stern 
layer, and consequently surface conduction only takes place in the diffuse layer. Revil and Glover (1998) stated that the electrical conductivity of rocks results from conduction through the bulk solution occupying the pores and from surface conduction occurring at the fluid/grain interface. Revil (1999) stated that the ionic diffusivities and the electrical conductivity in colloids and porous media receive contributions from diffusion and electromigration processes occurring in the bulk solution occupying the pores, plus electromigration occurring at the grain-water interface in the EDL. The author claimed that surface diffusion in porous materials presents no contribution from concentration gradients along the grain-water interface. Instead, surface diffusion is envisioned as a purely electromigration process due to the membrane potential.

\subsection{Porous Media and Homogenization Methods}

Recently, Volfkovich et al. (2013) reported a method for measuring the surface conductivity in porous electrodes. The authors defined surface conductivity as the tangential (longitudinal) conductivity of the EDL. Volfkovich et al. (2013) stated that surface conductivity comprises the conductivity induced by the conductivity of the counterions of the surface groups in a highly dispersed carbon electrode $\kappa_{\mathrm{sg}}$, the surface conductivity $\kappa_{\mathrm{sch}}$ induced by the electrostatic charge of the inner surface of the electrode pores, and the conductivity produced by the ions in the diffused part of the EDL. The authors proposed that the total conductivity varies linearly with the solution concentration for very dilute solutions. The limiting value as the solution concentration tends to zero is given by the surface conductivity.

Flow and transport processes in porous media occur on different spatial and temporal scales. Additionally, the structure of the porous medium itself generally shows a high dependence on the spatial scale (Niessner and Helming, 2007). The transmission of information from one length scale to another is accomplished by the use of a suitable homogenization method. To the best knowledge of the authors, no article in literature describes combined surface and bulk transport processes in charged porous media using an established homogenization technique. Several articles in literature have dealt with transport processes in charged porous media using homogenization techniques; however, these articles have assumed that surface transport processes can be neglected compared to bulk transport processes (Pivonka et. al., 2007; Pivonka et al. 2009; Scheiner et al., 2010; Biesheuvel and Bazant. 2010; Gabitto and Tsouris, 2015; among others).

Samson et al. (2005) developed a description of ionic transport in unsaturated porous materials due to gradients in the electrochemical potential and the moisture content by averaging the relevant microscopic transport equations over a representative volume element (REV). The authors derived a set of time-dependent equations for both the concentration of ionic species within the pore electrolyte and the moisture content within the pore space. Looker and Carnie (2006) studied the transport of an n-component electrolyte in a dilute solvent through a rigid, porous body subjected to an external electric field. The mathematical microscopic description was given by using the linearized ionic transport equations, including the effects of ion diffusion, electromigration, and convection. Explicit expressions for the transport coefficient tensors were obtained in terms of solutions to cell problems.

Revil and Linde (2006) determined the macroscopic transport properties of isotropic microporous media by volume-averaging the local Nernst-Planck and Navier-Stokes equations 
in non-isothermal conditions. Instead of using Poisson-Boltzmann distributions to describe the ionic concentrations in the pore space of the medium the authors used a Donnan distribution obtained by equating the chemical potentials of the water molecules and ions between a reservoir of ions and the pore space of the medium. Revil and Jougnot (2008) used the volume average of the Nernst-Planck equation to determine the constitutive equations for the coupled diffusion flux and current density of a multicomponent electrolyte in unsaturated porous conditions. The authors assumed that water is the wetting phase for the solid phase and that the porous medium was isotropic and rigid.

Smith and co-workers (Pivonka et. al., 2007; Pivonka et al. 2009; Mohajeri et al., 2009; Scheiner et al., 2010) have studied transport process in charged porous media using an upscaling scheme based upon the formulation of volume averaged Poisson-Nernst-Planck equations. The authors followed a volume averaging type of formulation; however, Pivonka et al. (2007) proposed the use of a novel intrinsic effective average, see theoretical section for more details, to satisfy the thermodynamic equilibrium condition given by the macroscopic Donnan equation. In the equilibrium equations derived by Pivonka et al. (2007) the (intrinsic) voltage relates to (intrinsic) effective ion concentrations. The intrinsic effective concentrations are, for ideal solutions, logarithmic volume averages of concentrations. Regarding the Poisson equation, the authors reported that the variation of the electric potential must be related to an effective fixed charge concentration.

Biesheuvel and Bazant (2010) presented a model for capacitive charging and desalination by ideally polarizable porous electrodes. This model excluded effects of Faradaic reactions or specific adsorption of ions. The authors discussed the theory for the case of a dilute, binary electrolyte using the Gouy-Chapman-Stern (GCS) model of the EDL. Gabitto and Tsouris (2015) used the physical model presented by Biesheuvel and Bazant (2010) to study the capacitive deionization process in homogeneous porous electrodes by means of a volume averaging method. The authors derived identical equations to the ones reported by Biesheuvel and Bazant (2010) plus an alternative formulation of the same problem. In both cases, surface transport processes were neglected compared to bulk transport processes. Schmuk and Bazant (2014) derived effective Poisson-Nernst-Planck (PNP) equations for macroscopic ion transport in charged porous media. A formal two-scale convergence method was used to derive effective equations valid at scales much larger than the geometrical period (Schmuck, 2011). Schmuk and Bazant (2014) reported that the transport coefficients (diffusivities and mobilities) become tensors related to the microstructure of the porous medium, and the effective permittivity is also a tensor. The homogenization process creates a new term in the Poisson equation, an average surface charge per unit volume. In the limit of thin EDLs, Poisson's equation is replaced by macroscopic electroneutrality (balancing ionic and surface charges). Sharma et al. (2015) derived similar homogenized equations using a volume averaging method (Whitaker, 1999). These authors also computed the value of the effective transport coefficients in isotropic porous media by solving the corresponding closure problems.

Ochoa-Tapia et al. (1993) presented the only study the authors were able to find in the literature including combined treatment of bulk and surface transport using a formal homogenization process. The authors studied bulk and surface diffusion in uncharged porous media using a volume averaging methodology (Whitaker, 1999). The authors studied a simple 
system where a linear isotherm was used to relate excess surface and bulk concentrations. In addition to the spatial-averaging theorem, the analysis requires the surface-averaging theorem, which was derived by the authors using standard vector analysis. Ochoa-Tapia (1993) calculated the value of the transport parameters in isotropic porous media by solving the appropriate closure problems in approximate, Chang type, unit cells of spatially periodic porous media.

The goal of this work is to derive the equations describing combined bulk and surface transport of ions in charged porous media using a volume averaging technique. We will derive the averaged equations and algebraically manipulate them for comparison with other authors. Finally, we will estimate the values of the transport coefficients appearing in the average equations.

\section{TheORETICAL DeVElopMENT}

\subsection{Model Description}

The physical model used in this work is similar to the one proposed by Biesheuvel and Bazant (2010) and used by Gabitto and Tsouris (2015) in their study of the CDI process neglecting surface transport. The porous electrode is divided into pore space filled with quasineutral electrolyte and a solid matrix. The solution inside the pore space exchanges ions with a charged, thin double-layer "skin" on the electrode matrix. This approach is based on Newman's macroscopic porous electrode theory (Johnson and Newman; 1971, Newman and Thomas-Alyea, 2004), with the same assumptions made. We will follow a route based on the volume averaging method (VAM) developed by Prof. S. Whitaker and co-workers over a period of many years (Whitaker, 1999). In the bulk of the quasi-neutral solution filling the pores, the Nernst-Planck (NP) equation is used (Pivonka et al., 2007, Pivonka et al., 2009, Scheiner et al., 2010). The ion flux of species $\mathrm{i}$ is given by:

$$
\underline{N_{i}}=-D_{i}\left\{\underline{\nabla} c_{i}+z_{i} c_{i} \underline{\nabla} \psi\right\},
$$

where $\mathrm{N}_{\mathrm{i}}$ is the ion flux, $\mathrm{c}_{\mathrm{i}}$ is the ion concentration, $\underline{\nabla}$ is the nabla operator, $\mathrm{z}_{\mathrm{i}}$ is the ion charge, $D_{i}$ is the diffusion coefficient of ionic species $i, \psi$ is the dimensionless electrostatic potential in the pores $\left(\phi / \mathrm{V}_{\mathrm{T}}\right), \phi$ is the electrostatic potential, and $\mathrm{V}_{\mathrm{T}}$ is the thermal voltage $\left(\mathrm{V}_{\mathrm{T}}=\mathrm{R} \mathrm{T} / \mathrm{F}\right)$. Equation (3) has been derived assuming that the isotropic mobility, $\mathrm{u}_{\mathrm{i}}$, is given by the NernstEinstein relation (Bockris et al., 2000) for constant absolute temperature $T\left(u_{i}=D_{i} / R T\right)$.

We consider a two-phase medium consisting of a $\alpha$ phase (liquid) and a $\beta$ phase (solid). The point species molar continuity equation, including the Nernst-Planck expression for the molar flux for the $\alpha$ phase, is given by:

$$
\partial \mathrm{c}_{\mathrm{i}} / \partial \mathrm{t}=-\underline{\nabla} \bullet \underline{\mathrm{N}_{\mathrm{i}}}
$$

Substituting eq. (3) into (4) yields:

$$
\partial \mathrm{c}_{\mathrm{i}} / \partial \mathrm{t}=\underline{\nabla} \bullet\left(\mathrm{D}_{\mathrm{i}}\left\{\underline{\nabla} \mathrm{c}_{\mathrm{i}}+\mathrm{z}_{\mathrm{i}} \mathrm{c}_{\mathrm{i}} \underline{\nabla} \psi\right\}\right)
$$

In the case of a monovalent ionic salt, $\mathrm{NaCl}$ for example, Eq. (5) leads to two differential equations for the positive and negative ions. Adding up these equations leads to a single equation in the salt concentration, $\mathrm{c}$, where the migration terms disappear if $\mathrm{c}_{-}=\mathrm{c}_{+}=\mathrm{c}$ and $\mathrm{D}=\mathrm{D}_{-}=\mathrm{D}_{+}$:

$$
\partial \mathrm{c} / \partial \mathrm{t}=\underline{\nabla} \bullet(\mathrm{D} \underline{\nabla} \mathrm{c})
$$

In order to calculate the dimensionless electrostatic potential in the system $(\psi)$, we will follow Gabitto and Tsouris (2015) who used a local charge balance in the bulk fluid for a quasineutral binary electrolyte to obtain: 


$$
\underline{\nabla} \bullet\left(\underline{\mathrm{I}}_{\mathrm{e}} / \mathrm{F}\right)=-\underline{\nabla} \bullet(\kappa \underline{\nabla} \psi)=-\underline{\nabla} \bullet(\mathrm{D} \mathrm{c} \underline{\nabla} \psi)=0
$$

Here, $\underline{I}_{\mathrm{e}}$ is the current carried by the ions per unit area, $\mathrm{F}$ is the Faraday constant, and $\kappa$ is the effective conductivity of the solution phase.

In order to explicitly express the surface charge density (q) and the surface excess neutral salt concentration (w) in terms of $\mathrm{c}$ and $\psi$, we use the GCS model (Bazant et al., 2004; Chu and Bazant, 2006; Zhao et al., 2010) that leads to:

$$
\begin{aligned}
\mathrm{w} & =8 \lambda_{\mathrm{D}} \mathrm{C}_{\infty} \sqrt{\mathrm{c} / \mathrm{C}_{\infty}} \sinh ^{2}\left(\Delta \psi_{\mathrm{D}} / 4\right), \\
\text { and } \mathrm{q} & =-4 \lambda_{\mathrm{D}} \mathrm{C}_{\infty} \sqrt{\mathrm{c} / \mathrm{C}_{\infty}} \sinh \left(\Delta \psi_{\mathrm{D}} / 2\right),
\end{aligned}
$$

where $\mathrm{C}_{\infty}$ is the bulk concentration, $\Delta \psi_{\mathrm{D}}$ is the potential difference over the diffuse layer, $\left(\Delta \psi_{\mathrm{D}}=\right.$ $\left.\psi_{\text {Stern }}-\psi\right), \psi_{\text {Stern }}$ is the Stern dimensionless potential, $\lambda_{\mathrm{D}}$ is the Debye length $\left(\lambda_{\mathrm{D}}=\right.$ $1 / \sqrt{8 \pi \lambda_{\mathrm{B}} \mathrm{N}_{\mathrm{av}} \mathrm{C}_{\infty}}$ ), $\mathrm{N}_{\mathrm{av}}$ is the Avogadro number, and $\lambda_{\mathrm{B}}$ (the Bjerrum length) is $\sim 0.72 \mathrm{~nm}$ at room temperature.

In this work, we will use the boundary conditions at the liquid-solid interface $\left(\mathrm{A}_{\alpha \beta}\right)$ reported by Chu and Bazant (2007) in the case of microscopically diffuse interfaces. The authors derived surface conservation laws for diffusive and electrochemical transport in terms of the electrochemical potential. In the case of dilute electrolytes, the electrochemical potential of the ionic species $\mathrm{i}\left(\mu_{\mathrm{i}}\right)$ is given by:

$$
\mu_{\mathrm{i}}=\mathrm{R} \mathrm{T} \ln \mathrm{c}_{\mathrm{i}}+\mathrm{z}_{\mathrm{i}} \mathrm{R} \mathrm{T} \psi
$$

Combination of the surface conservation laws for individual ions in the case of a symmetric, binary electrolyte leads to the following equations in terms of the surface charge density (q) and the surface excess neutral salt concentration (w) as:

$$
\begin{aligned}
& \frac{\partial \mathrm{w}}{\partial \mathrm{t}}=\underline{\nabla}_{\mathrm{s}} \bullet\left\{\mathrm{D}_{\mathrm{s}}\left[\mathrm{w} \underline{\nabla}_{\mathrm{s}} \ln (\mathrm{c})+\mathrm{z}_{+} \mathrm{q} \underline{\nabla}_{\mathrm{s}} \psi\right]\right\}-\underline{\mathrm{n}}_{\alpha \beta} \bullet(\mathrm{D} \underline{\nabla} \mathrm{c}), \\
& \frac{\partial \mathrm{q}}{\partial \mathrm{t}}=\underline{\nabla}_{\mathrm{s}} \bullet\left\{\mathrm{D}_{\mathrm{s}}\left[\mathrm{q} \underline{\nabla}_{\mathrm{s}} \ln (\mathrm{c})+\mathrm{z}_{+} \mathrm{w} \underline{\nabla}_{\mathrm{s}} \psi\right]\right\}-\underline{\mathrm{n}}_{\alpha \beta} \bullet(\mathrm{D} \mathrm{c} \underline{\nabla} \psi),
\end{aligned}
$$

where $\underline{\mathrm{n}}_{\alpha \underline{\beta}}$ is the unit outwardly directed normal vector pointing from the $\alpha$-phase into the $\beta$ phase, $z_{+}$is the absolute value of the ion charge, $D_{s}$ is the salt surface diffusivity, $\underline{\nabla}_{s}$ is the surface gradient operator $\left(\underline{\nabla}_{\mathrm{s}}=\underline{\underline{\mathrm{P}}} \bullet \underline{\nabla}\right)$, and $\underline{\underline{\mathrm{P}}}$ is the projection tensor for the $\alpha-\beta$ interface $\left(\underline{\underline{\mathrm{P}}}=\underline{\underline{\mathbf{I}}}-\underline{\mathbf{n}}_{\alpha \beta} \underline{\mathbf{n}}_{\alpha \beta}\right)$.

Biesheuvel and Bazant (2010) and Gabitto and Tsouris (2015) used similar boundary conditions. However, in both cases the authors did not consider the influence of the surface transport terms by neglecting the first term in the right-hand-side compared to the second.

\subsection{Species Continuity Equation}

We start our derivation from Eq. (6) and the boundary condition at the $\alpha-\beta$ interphase given by Eq. (11). The boundary conditions in the input-output area of the macroscopic domain are not relevant to the volume averaging method, as several authors (Ryan et al., 1980; Carbonell and Whitaker, 1984; Nozad et al., 1985; Whitaker, 1986; Quintard and Whitaker, 1998; Ulson de Souza and Whitaker, 2003) have proved that the problem under study can be always expressed by a local problem that does not require knowledge of these boundary conditions. The physical system defined by equations (6) and (11) is somewhat similar to the diffusion with surface 
adsorption problem studied by several authors (Quintard and Whitaker, 1998, Ulson de Souza and Whitaker, 2003). The source term enters into the volume averaged equation through the boundary condition.

A variety of averages is encountered in this type of analysis (Whitaker, 1999). The traditionally encountered averages are the phase average given by:

$$
\left\langle\mathrm{c}_{\alpha}\right\rangle=\frac{1}{\mathrm{~V}} \int_{\mathrm{V}} \mathrm{c}_{\alpha} \mathrm{dV}
$$

and the intrinsic phase average which takes the form:

$$
\left\langle\mathrm{c}_{\alpha}\right\rangle^{\alpha}=\frac{1}{\mathrm{~V}_{\alpha}} \int_{\mathrm{V}} \mathrm{c}_{\alpha} \mathrm{dV}=\varepsilon_{\alpha}\left\langle\mathrm{c}_{\alpha}\right\rangle \text {. }
$$

Here, $\mathrm{V}$ is the volume of the $\mathrm{REV}, \mathrm{V}_{\alpha}$ represents the volume of the $\alpha$ phase contained within the $\mathrm{REV}$, and $\varepsilon_{\alpha}$ is the volume fraction given explicitly by $\varepsilon_{\alpha}=\mathrm{V}_{\alpha} / \mathrm{V}$.

Recently, several authors (Pivonka et. al., 2007; Pivonka et al. 2009; Scheiner et al., 2010) have proposed the use of an intrinsic effective average, $\left\langle\widehat{\mathrm{c}}_{\alpha}\right\rangle^{\alpha}$, defined as:

$$
\left\langle\widehat{\mathrm{c}}_{\alpha}\right\rangle^{\alpha}=\operatorname{Exp}\left\{\left\langle\ln \mathrm{c}_{\alpha}\right\rangle^{\alpha}\right\}=\operatorname{Exp}\left\{\frac{1}{\mathrm{~V}_{\alpha}} \int_{\mathrm{V}} \ln \mathrm{c}_{\alpha} \mathrm{dV}\right\}
$$

We also use the spatial averaging theorem (Gray and Lee, 1977) that takes the form for a scalar variable $\psi_{\alpha}$ :

$$
\left\langle\underline{\nabla} \psi_{\alpha}\right\rangle=\underline{\nabla}\left\langle\psi_{\alpha}\right\rangle+\frac{1}{\mathrm{~V}} \int_{\mathrm{A}_{\alpha \beta}} \underline{\mathrm{n}}_{\alpha \beta} \psi_{\alpha} \mathrm{dA} .
$$

In the study of surface transport, it is also necessary to use the surface averaging theorem presented by Ochoa-Tapia et al. (1993) as:

$$
\int_{\mathrm{A}_{\alpha \beta}} \underline{\nabla}_{\mathrm{s}} \psi_{\alpha} \mathrm{dA}=\underline{\nabla} \int_{\mathrm{A}_{\alpha \beta}} \psi_{\alpha} \mathrm{dA}+\int_{\mathrm{A}_{\alpha \beta}} 2 \mathrm{H}_{\alpha \beta} \underline{\mathrm{n}}_{\alpha \beta} \psi_{\alpha} \mathrm{dA} .
$$

Here, $\mathrm{H}$ is the curvature calculated by:

$$
2 \mathrm{H}=\underline{\nabla}_{\mathrm{s}} \bullet \underline{\mathrm{n}}_{\alpha \beta} \text {. }
$$

After repeated use of the averaging tools described above, plus algebraic manipulations, we obtain the following volume averaged equation:

$$
\begin{aligned}
& \varepsilon_{\alpha} \frac{\partial \mathrm{c}\rangle^{\alpha}}{\partial \mathrm{t}}=\underline{\nabla} \bullet\left\{\varepsilon_{\alpha} \mathrm{D}\left[\underline{\nabla}\langle\mathrm{c}\rangle^{\alpha}+\frac{1}{\mathrm{~V}_{\alpha}} \int_{\mathrm{A}_{\alpha \beta}} \underline{\mathrm{n}}_{\alpha \beta} \tilde{\mathrm{c}} \mathrm{dA}\right]\right\}-\mathrm{a}_{\mathrm{v}} \frac{\partial \mathrm{w}\rangle_{\alpha \beta}}{\partial \mathrm{t}}+\frac{1}{\mathrm{~V}} \int_{\mathrm{A}_{\alpha \beta}} \underline{\nabla}_{\mathrm{s}} \bullet \mathrm{D}_{\mathrm{s}}\left[\frac{\mathrm{W}}{\mathrm{c}} \underline{\nabla}_{\mathrm{s}} \mathrm{c}\right] \mathrm{dA} \\
& +\frac{1}{\mathrm{~V}} \int_{\mathrm{A}_{\alpha \beta}} \underline{\nabla}_{\mathrm{s}} \bullet \mathrm{D}_{\mathrm{s}}\left[\mathrm{z}_{+} \mathrm{q} \underline{\nabla}_{\mathrm{s}} \psi \mathrm{dA}\right] \mathrm{dA} .
\end{aligned}
$$

The last two terms on the right-hand side of Eq. (19) represent the contributions of surface transport processes. In the derivation of Eq. (19), we have used Gray's decomposition for the salt concentration (Gray, 1975):

$$
\mathrm{c}=\langle\mathrm{c}\rangle^{\alpha}+\tilde{\mathrm{c}} \text {. }
$$

Two consecutive applications of the surface averaging theorem, Eq. (17), plus use of the following assumptions: $\mathrm{q} \cong\langle\mathrm{q}\rangle_{\alpha \beta}, \mathrm{w} / \mathrm{c} \cong\langle\mathrm{w}\rangle_{\alpha \beta} /\langle\mathrm{c}\rangle^{\alpha}$ lead to: 


$$
\begin{aligned}
& \varepsilon_{\alpha} \frac{\partial \mathrm{c}\rangle^{\alpha}}{\partial \mathrm{t}}=\underline{\nabla} \bullet\left\{\varepsilon_{\alpha} \mathrm{D}\left[\underline{\nabla}\langle\mathrm{c}\rangle^{\alpha}+\frac{1}{\mathrm{~V}_{\alpha}} \int_{\mathrm{A}_{\alpha \beta}} \underline{\mathrm{n}}_{\alpha \beta} \tilde{\mathrm{c}} \mathrm{dA}\right]\right\}-\mathrm{a}_{\mathrm{v}} \frac{\partial \mathrm{w}\rangle_{\alpha \beta}}{\partial \mathrm{t}} \\
& +\frac{1}{\mathrm{~V}} \underline{\nabla} \bullet\left[\mathrm{D}_{\mathrm{s}} \frac{\langle\mathrm{w}\rangle_{\alpha \beta}}{\langle\mathrm{c}\rangle^{\alpha}} \int_{\mathrm{A}_{\alpha \beta}} \underline{\nabla}_{\mathrm{s}} \mathrm{cdA}\right]+\frac{1}{\mathrm{~V}} \mathrm{D}_{\mathrm{s}} \frac{\langle\mathrm{w}\rangle_{\alpha \beta}}{\langle\mathrm{c}\rangle^{\alpha}} \int_{\mathrm{A}_{\alpha \beta}} 2 \mathrm{H} \underline{\mathrm{n}}_{\alpha \beta} \bullet \underline{\nabla}_{\mathrm{s}} \mathrm{cdA} \\
& +\mathrm{z}_{+} \frac{1}{\mathrm{~V}} \underline{\nabla} \bullet \mathrm{D}_{\mathrm{s}}\langle\mathrm{q}\rangle_{\alpha \beta} \int_{\mathrm{A}_{\alpha \beta}} \underline{\nabla}_{\mathrm{s}} \psi \mathrm{dA}+\mathrm{z}_{+} \frac{1}{\mathrm{~V}} \mathrm{D}_{\mathrm{s}}\langle\mathrm{q}\rangle_{\alpha \beta} \int_{\mathrm{A}_{\alpha \beta}} 2 \mathrm{H} \underline{\mathrm{n}}_{\alpha \beta} \bullet \underline{\nabla}_{\mathrm{s}} \psi \mathrm{dA}
\end{aligned}
$$

Here, $\langle\mathrm{q}\rangle_{\alpha \beta}$ and $\langle\mathrm{w}\rangle_{\alpha \beta}$ are the excess salt adsorption and the surface charge density averaged at the interface area $\alpha-\beta$, respectively, and calculated by:

$$
\langle\mathrm{w}\rangle_{\alpha \beta}=\frac{1}{\mathrm{~A}_{\alpha \beta}} \int_{\mathrm{A}_{\alpha \beta}} \mathrm{wdA},\langle\mathrm{q}\rangle_{\alpha \beta}=\frac{1}{\mathrm{~A}_{\alpha \beta}} \int_{\mathrm{A}_{\alpha \beta}} \mathrm{qdA} .
$$

Complex algebraic manipulations of eq. (21) lead to,

$$
\begin{aligned}
& \varepsilon_{\alpha} \frac{\partial\langle\mathrm{c}\rangle^{\alpha}}{\partial \mathrm{t}}=\underline{\nabla} \bullet\left\{\varepsilon_{\alpha} \mathrm{D}\left[\underline{\nabla}\langle\mathrm{c}\rangle^{\alpha}+\frac{1}{\mathrm{~V}_{\alpha}} \int_{\mathrm{A}_{\alpha \beta}} \underline{\mathrm{n}}_{\alpha \beta} \tilde{\mathrm{c}} \mathrm{dA}\right]\right\}-\mathrm{a}_{\mathrm{v}} \frac{\partial \mathrm{w}\rangle_{\alpha \beta}}{\partial \mathrm{t}}+\underline{\nabla} \bullet\left\{\mathrm { a } _ { \mathrm { v } } \mathrm { D } _ { \mathrm { s } } \frac { \langle \mathrm { w } \rangle _ { \alpha \beta } } { \langle \mathrm { c } \rangle ^ { \alpha } } \left[\underline{\nabla}\langle\mathrm{c}\rangle^{\alpha}\right.\right. \\
& \left.\left.+\frac{1}{\mathrm{~A}_{\alpha \beta}} \int_{\mathrm{A}_{\alpha \beta}} 2 \mathrm{H} \underline{\mathrm{n}}_{\alpha \beta} \tilde{\mathrm{c}} \mathrm{dA}\right]\right\}+\mathrm{z}_{+} \underline{\nabla} \bullet\left\{\mathrm{a}_{\mathrm{v}} \mathrm{D}_{\mathrm{s}}\langle\mathrm{q}\rangle_{\alpha \beta}\left[\underline{\nabla}\langle\psi\rangle^{\alpha}+\frac{1}{\mathrm{~A}_{\alpha \beta}} \int_{\mathrm{A}_{\alpha \beta}} 2 \mathrm{H} \underline{\mathrm{n}}_{\alpha \beta} \tilde{\psi} \mathrm{dA}\right]\right\}
\end{aligned}
$$

Here, $a_{v}$ is the effective area $\left(A_{\alpha \beta} / V\right)$. In the derivation of Eq. (23), we have used the fact that $\int_{\mathrm{A}_{\alpha \beta}} 2 \mathrm{H} \underline{\mathrm{n}}_{\alpha \beta} \bullet \underline{\nabla}_{\mathrm{s}} \mathrm{c} \mathrm{dA}=\int_{\mathrm{A}_{\alpha \beta}} 2 \mathrm{H} \underline{\mathrm{n}}_{\alpha \beta} \bullet \underline{\nabla}_{\mathrm{s}} \psi \mathrm{dA}=0$, since $\underline{\mathrm{n}}_{\alpha \beta}$ is orthogonal to the surface gradient. We also used the assumption, $\langle\mathrm{c}\rangle_{\alpha \beta} \cong\langle\mathrm{c}\rangle^{\alpha},\langle\psi\rangle_{\alpha \beta} \cong\langle\psi\rangle^{\alpha}$, and Gray's decomposition for the dimensionless potential,

$$
\psi=\langle\psi\rangle^{\alpha}+\tilde{\psi} \text {. }
$$

Equation (23) cannot be closed without introducing constitutive expressions for the scalar deviation fields $\tilde{c}$ and $\tilde{\psi}$. This task will be carried out in the following sections.

\subsubsection{Closure for Species Continuity Equation}

We start our derivation from the boundary condition at the $\alpha-\beta$ interface given by Eq. (11). After algebraic manipulations, we obtain:

$$
\begin{aligned}
& \underline{\mathrm{n}}_{\alpha \beta} \bullet(\mathrm{D} \underline{\nabla} \tilde{\mathrm{c}})-\underline{\nabla}_{\mathrm{s}} \bullet\left[\mathrm{D}_{\mathrm{s}} \frac{\langle\mathrm{w}\rangle_{\alpha \beta}}{\langle\mathrm{c}\rangle^{\alpha}} \underline{\nabla}_{\mathrm{s}} \tilde{\mathrm{c}}\right]=\underline{\nabla}_{\mathrm{s}} \bullet\left[\mathrm{D}_{\mathrm{s}} \frac{\langle\mathrm{w}\rangle_{\alpha \beta}}{\langle\mathrm{c}\rangle^{\alpha}} \underline{\nabla}_{\mathrm{s}}\langle\mathrm{c}\rangle^{\alpha}\right] \\
& +\mathrm{z}_{+} \underline{\nabla}_{\mathrm{s}} \bullet\left[\mathrm{D}_{\mathrm{s}}\langle\mathrm{q}\rangle_{\alpha \beta} \underline{\nabla}_{\mathrm{s}} \psi\right]-\underline{\mathrm{n}}_{\alpha \beta} \bullet\left(\mathrm{D} \underline{\nabla}\langle\mathrm{c}\rangle^{\alpha}\right)-\frac{\partial\langle\mathrm{w}\rangle_{\alpha \beta}}{\partial \mathrm{t}}
\end{aligned}
$$

The first and second terms in the right-hand-side of Eq. (25) can be expanded using the relationship between surface Laplacian and averaged variables presented by Ochoa-Tapia et al. (1993), for example:

$$
\underline{\nabla}_{\mathrm{s}} \bullet \underline{\nabla}_{\mathrm{s}}\langle\mathrm{c}\rangle^{\alpha}=\underline{\nabla}^{2}\langle\mathrm{c}\rangle^{\alpha}-\left(\underline{\mathrm{n}}_{\alpha \beta} \underline{\mathrm{n}}_{\alpha \beta}\right): \underline{\nabla} \underline{\nabla}\langle\mathrm{c}\rangle^{\alpha}-2 \mathrm{H} \underline{\mathrm{n}}_{\alpha \beta} \bullet \underline{\nabla}\langle\mathrm{c}\rangle^{\alpha}
$$


Introducing Eq. (26) into Eq. (21) will produce an expression with 8 different sources. Obviously, this complex equation will be very hard to handle. Ochoa-Tapia et al. (1993) encountered the same problem in the simpler study of surface diffusion in uncharged porous media. At this point, we follow the same rout presented by Ochoa-Tapia et al. (1993) proposing several constraints that will significantly simplify the mathematical complexity of the problem.

$$
\left(\frac{\langle\mathrm{w}\rangle_{\alpha \beta}}{\langle\mathrm{c}\rangle^{\alpha} \mathrm{L}_{\mathrm{c}}}\right)<<1,\left(\frac{\langle\mathrm{q}\rangle_{\alpha \beta}\langle\psi\rangle^{\alpha}}{\langle\mathrm{c}\rangle^{\alpha} \mathrm{L}_{\mathrm{c}}}\right)<<1,\left(\frac{\mathrm{D}_{\mathrm{s}}\langle\mathrm{w}\rangle_{\alpha \beta}}{\mathrm{D}\langle\mathrm{c}\rangle^{\alpha} \mathrm{L}_{\mathrm{c}}}\right)<<1,\left(\frac{\mathrm{D}_{\mathrm{s}}\langle\mathrm{q}\rangle_{\alpha \beta}\langle\psi\rangle^{\alpha}}{\mathrm{D}\langle\mathrm{c}\rangle^{\alpha} \mathrm{L}_{\mathrm{c}}}\right)<<1 .
$$

Here, $\mathrm{L}_{\mathrm{c}}$ is a characteristic macroscopic length. After introduction of Eq. (26) into Eq. (25) and algebraic manipulations, the use of the constraints given by Eq. (27) leads to a much simplified problem given by:

$$
\begin{aligned}
& \underline{\mathrm{n}}_{\alpha \beta} \bullet(\mathrm{D} \underline{\nabla} \tilde{\mathrm{c}})-\underline{\nabla}_{\mathrm{s}} \bullet\left[\mathrm{D}_{\mathrm{s}} \frac{\langle\mathrm{w}\rangle_{\alpha \beta}}{\langle\mathrm{c}\rangle^{\alpha}} \underline{\nabla}_{\mathrm{s}} \tilde{\mathrm{c}}\right]=-\underline{\mathrm{n}}_{\alpha \beta} \bullet\left[2 \mathrm{H} \mathrm{D}_{\mathrm{s}} \frac{\langle\mathrm{w}\rangle_{\alpha \beta}}{\langle\mathrm{c}\rangle^{\alpha}}+\mathrm{D}\right] \underline{\nabla}\langle\mathrm{c}\rangle^{\alpha} \\
& -\mathrm{z}_{+} 2 \mathrm{H} \underline{\mathrm{n}}_{\alpha \beta} \bullet\left[\mathrm{D}_{\mathrm{s}}\langle\mathrm{q}\rangle_{\alpha \beta} \underline{\nabla} \psi\right]-\frac{\partial\langle\mathrm{w}\rangle_{\alpha \beta}}{\partial \mathrm{t}} .
\end{aligned}
$$

Therefore, we propose that:

$$
\left.\tilde{\mathrm{c}}=\underline{\mathrm{f}}_{1} \bullet \underline{\nabla}\langle\mathrm{c}\rangle^{\alpha}+\underline{\mathrm{f}}_{2} \bullet \underline{\nabla}\langle\psi\rangle^{\alpha}+\mathrm{f}_{3} \partial \mathrm{w}\right\rangle_{\alpha \beta} / \partial \mathrm{t} .
$$

We obtain the equation for the salt concentration deviation ( $\tilde{\mathrm{c}}$ ) by subtracting Eq. (19) divided by $\varepsilon_{\alpha}$ from Eq. (4) to obtain:

$$
\frac{\partial \tilde{\mathrm{c}}}{\partial \mathrm{t}}=\underline{\nabla} \bullet(\mathrm{D} \underline{\nabla} \tilde{\mathrm{c}})-\underline{\nabla} \bullet\left(\frac{\mathrm{a}_{\mathrm{v}}}{\varepsilon_{\alpha}} \mathrm{D}_{\mathrm{s}} \frac{\langle\mathrm{w}\rangle_{\alpha \beta}}{\langle\mathrm{c}\rangle^{\alpha}} \underline{\nabla}\langle\mathrm{c}\rangle^{\alpha}\right)-\underline{\nabla} \bullet\left(\frac{\mathrm{a}_{\mathrm{v}}}{\varepsilon_{\alpha}} \mathrm{D}_{\mathrm{s}}\langle\mathrm{q}\rangle_{\alpha \beta} \underline{\nabla}\langle\psi\rangle^{\alpha}\right)+\frac{\mathrm{a}_{\mathrm{v}}}{\varepsilon_{\alpha}} \frac{\partial \mathrm{w}\rangle_{\alpha \beta}}{\partial \mathrm{t}} .
$$

An order of magnitude analysis shows that the integral terms are negligible compared to the first on the right-hand-side of Eq. (30). The second and third terms are also negligible due to the constraints given in Eq. (27). The local process in the REV is quasi-stationary when the following restriction holds:

$$
\mathrm{t}^{*} \mathrm{D} / \mathrm{l}_{\alpha}^{2} \gg 1 \text {. }
$$

Here $t^{*}$ is the characteristic time for the mass transport process and $l_{\alpha}$ is a characteristic length of the microscale. According to Biesheuvel and Bazant (2010), the characteristic time for the supercapacitor regime $\left(\mathrm{t}_{\mathrm{C}}^{\bullet}\right)$ and the characteristic time for the desalination regime $\left(\mathrm{t}_{\mathrm{D}}^{\bullet}\right)$ both satisfy the condition given by Eq. (31). In conclusion, the equation to calculate the salt concentration deviations is reduced to:

$$
\underline{\nabla} \bullet(\mathrm{D} \underline{\nabla} \tilde{\mathrm{c}})=-\frac{\mathrm{a}_{\mathrm{v}}}{\varepsilon_{\alpha}} \frac{\partial \mathrm{w}\rangle_{\alpha \beta}}{\partial \mathrm{t}} .
$$

The $\underline{\mathrm{f}}_{1}$ and $\underline{\mathrm{f}}_{2}$ vector fields, and the scalar $\mathrm{f}_{3}$ field closure parameters are calculated by solving the following boundary-value problems:

\section{Problem 1}

$$
\underline{\nabla}^{2} \underline{\mathrm{f}}_{1}=0
$$




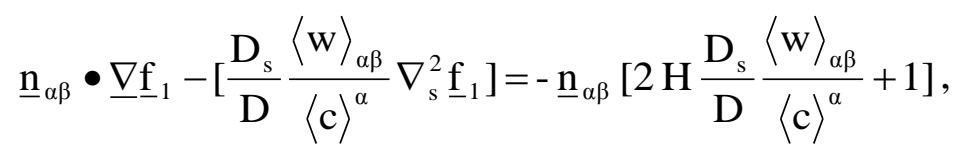

at the $A_{\alpha \beta}$ interface and for spatially periodic porous media:

$$
\underline{\mathrm{f}}_{1}\left(\underline{\mathrm{r}}+\underline{\mathrm{l}}_{\mathrm{i}}\right)=\underline{\mathrm{f}}_{1}(\underline{\mathrm{r}}) \text {. }
$$

Problem 2

$$
\begin{aligned}
& \underline{\nabla}^{2} \underline{\mathrm{f}}_{2}=0 \\
& \underline{\mathrm{n}}_{\alpha \beta} \bullet\left(\underline{\nabla}_{2}\right)-\left[\frac{\mathrm{D}_{\mathrm{s}}}{\mathrm{D}} \frac{\langle\mathrm{w}\rangle_{\alpha \beta}}{\langle\mathrm{c}\rangle^{\alpha}} \underline{\nabla}_{\mathrm{s}}^{2} \underline{\mathrm{f}}_{2}\right]=-\mathrm{z}_{+} 2 \mathrm{H} \underline{\mathrm{n}}_{\alpha \beta} \frac{\mathrm{D}_{\mathrm{s}}\langle\mathrm{q}\rangle_{\alpha \beta}}{\mathrm{D}\langle\mathrm{c}\rangle^{\alpha}},
\end{aligned}
$$

at the $A_{\alpha \beta}$ interface and for spatially periodic porous media:

$$
\underline{\mathrm{f}}_{2}\left(\underline{\mathrm{r}}+\underline{\mathrm{l}}_{\mathrm{i}}\right)=\underline{\mathrm{f}}_{2}(\underline{\mathrm{r}}) \text {. }
$$

\section{Problem 3}

$$
\begin{aligned}
& \underline{\nabla}^{2} \mathrm{f}_{2}=-\mathrm{a}_{\mathrm{v}} /\left(\varepsilon_{\alpha} \mathrm{D}\right), \\
& \underline{\mathrm{n}}_{\alpha \beta} \underline{\nabla} \mathrm{f}_{3}-\frac{\mathrm{D}_{\mathrm{s}}}{\mathrm{D}} \frac{\langle\mathrm{w}\rangle_{\alpha \beta}}{\langle\mathrm{c}\rangle^{\alpha}} \nabla_{\mathrm{s}}^{2} \mathrm{f}_{3}=-\frac{1}{\mathrm{D}},
\end{aligned}
$$

at the $A_{\alpha \beta}$ interface and also for spatially periodic porous media:

$$
\mathrm{f}_{3}\left(\underline{\mathrm{r}}+\underline{\mathrm{l}}_{\mathrm{i}}\right)=\mathrm{f}_{3}(\underline{\mathrm{r}}) \text { for } \mathrm{i}=1,2,3 .
$$

Here, and in equations (35) and (38), $\underline{l}_{i}$ represents the three non-unique lattice vectors that are needed to describe a spatially periodic porous medium (Brenner, 1980).

\subsubsection{Closed Species Continuity Equation}

The closed species continuity equation is obtained by replacing the salt concentration deviations ( $\tilde{\mathrm{c}}$ ) and electrical potential deviations ( $\tilde{\psi})$ in Eq. (19) by equations (29) and (51), respectively. After complex algebraic manipulations we obtain:

$$
\begin{aligned}
& \varepsilon_{\alpha} \frac{\partial \mathrm{c}\rangle^{\alpha}}{\partial \mathrm{t}}=\underline{\nabla} \bullet\left\{\varepsilon_{\alpha} \underline{\mathrm{D}}_{\text {eff }} \bullet \underline{\nabla}\langle\mathrm{c}\rangle^{\alpha}\right\}+\underline{\nabla} \bullet\left\{\mathrm{a}_{\mathrm{v}} \underline{\underline{D}}_{\text {eff }}^{\mathrm{s}} \bullet \frac{\langle\mathrm{w}\rangle_{\alpha \beta}}{\langle\mathrm{c}\rangle^{\alpha}} \underline{\nabla}\langle\mathrm{c}\rangle^{\alpha}\right\} \\
& +\mathrm{z}_{+} \underline{\nabla} \bullet\left\{\mathrm{a}_{\mathrm{v}} \underline{\underline{\mathrm{D}}}_{\mathrm{eff}}^{\mathrm{m}} \bullet\langle\mathrm{q}\rangle_{\alpha \beta} \underline{\nabla}\langle\psi\rangle^{\alpha}\right\}-\mathrm{a}_{\mathrm{v}} \frac{\partial \mathrm{w}\rangle_{\alpha \beta}}{\partial \mathrm{t}}
\end{aligned}
$$

The nontraditional terms have been eliminated using the constraints given by Eq. (27). The bulk effective diffusivity tensor $\left(\underline{\underline{D}}_{\text {eff }}\right)$, the surface effective diffusivity tensor $\left(\underline{\underline{D}}_{\text {eff }}^{\mathrm{s}}\right)$, and the surface effective mobility tensor $\left(\underline{\underline{D}}_{\text {eff }}^{\mathrm{m}}\right)$ are given by:

$$
\begin{aligned}
& \underline{\underline{D}}_{\text {eff }}=\mathrm{D}\left(\underline{\underline{\mathrm{I}}}+\frac{1}{\mathrm{~V}_{\alpha}} \int_{\mathrm{A}_{\alpha \beta}} \underline{\mathrm{n}}_{\alpha \beta} \underline{\mathrm{f}}_{1} \mathrm{dA}\right), \\
& \underline{\underline{\mathrm{D}}}_{\mathrm{eff}}^{\mathrm{s}}=\mathrm{D}_{\mathrm{s}}\left[\underline{\underline{\mathrm{I}}}+\frac{1}{\mathrm{~A}_{\alpha \beta}} \int_{\mathrm{A}_{\alpha \beta}} 2 \mathrm{H} \underline{\underline{n}}_{\alpha \beta} \underline{\mathrm{f}}_{1} \mathrm{dA}+\mathrm{z}_{+} \frac{\langle\mathrm{c}\rangle^{\alpha}\langle\mathrm{q}\rangle_{\alpha \beta}}{\langle\mathrm{w}\rangle_{\alpha \beta}} \frac{1}{\mathrm{~A}_{\alpha \beta}} \int_{\mathrm{A}_{\alpha \beta}} 2 \mathrm{H} \underline{\mathrm{n}}_{\alpha \beta} \underline{\mathrm{g}}_{1} \mathrm{dA}\right],
\end{aligned}
$$




$$
\begin{aligned}
& \underline{\mathrm{D}}_{\mathrm{eff}}^{\mathrm{m}}=\mathrm{D}_{\mathrm{s}}\left[\mathrm{I}+\frac{1}{\mathrm{~A}_{\alpha \beta}} \int_{\mathrm{A}_{\alpha \beta}} 2 \mathrm{H} \underline{\mathrm{n}}_{\alpha \beta} \underline{\mathrm{g}}_{2} \mathrm{dA}+\frac{\langle\mathrm{w}\rangle_{\alpha \beta}}{\langle\mathrm{q}\rangle_{\alpha \beta}\langle\mathrm{c}\rangle^{\alpha}} \frac{1}{\mathrm{~A}_{\alpha \beta}} \int_{\mathrm{A}_{\alpha \beta}} 2 \mathrm{H} \underline{\mathrm{n}}_{\alpha \beta} \underline{\mathrm{f}}_{2} \mathrm{dA}\right. \\
& \left.+\frac{\mathrm{D}\langle\mathrm{c}\rangle^{\alpha}}{2 \mathrm{H} \mathrm{D}_{\mathrm{s}}\langle\mathrm{q}\rangle_{\alpha \beta}\langle\mathrm{c}\rangle^{\alpha}} \frac{2 \mathrm{H}}{\mathrm{A}_{\alpha \beta}} \int_{\mathrm{A}_{\alpha \beta}} \underline{\mathrm{n}}_{\alpha \beta} \underline{\mathrm{f}}_{2} \mathrm{dA}\right]
\end{aligned}
$$

In Eq. (42), the first term on the right-hand-side represents mainly bulk diffusion while the second and third terms represent surface diffusion and migration, respectively. The last term on the right-hand-side represents ion accumulation in the EDLs. Inspection of equations (43) shows that the calculation of the effective diffusivities requires the solution of problems 1 and 2 .

If the surface transport terms are negligible compared to bulk diffusion, we obtain the following equation:

$$
\varepsilon_{\alpha} \partial\left\langle\mathrm{c}_{\alpha}\right\rangle^{\alpha} / \partial \mathrm{t}=\underline{\nabla} \bullet\left(\varepsilon_{\alpha} \underline{\underline{D}}_{\text {eff }} \bullet \underline{\nabla}\left\langle\mathrm{c}_{\alpha}\right\rangle^{\alpha}\right)-\mathrm{a}_{\mathrm{v}} \partial\langle\mathrm{w}\rangle_{\alpha \beta} / \partial \mathrm{t} .
$$

Eq. (44) is identical to the equation originally proposed by Biesheuvel and Bazant (2010) and the one derived by Gabitto and Tsouris (2015) using the volume averaging method. In both cases, the authors started their derivations neglecting surface transport processes. However, the effective diffusivity calculated using Eq. (43a) will not be equal to the one calculated by Gabitto and Tsouris (2015). More will be said about this issue in the results section. Eq. (44) can also be reduced to Eq. 4.34, presented by Ochoa-Tapia et al. (1993), if we allow for the differences in treatment of the $\langle\mathrm{w}\rangle_{\alpha \beta} /\langle\mathrm{c}\rangle^{\alpha}$ ratio.

We can also follow Ochoa-Tapia et al. (1993) by combining bulk and surface diffusion into a single global term. After some algebraic manipulations we obtain:

$$
\varepsilon_{\alpha} \frac{\partial \mathrm{c}\rangle^{\alpha}}{\partial \mathrm{t}}=\underline{\nabla} \bullet\left\{\varepsilon_{\alpha} \underline{\underline{D}}^{*} \bullet \underline{\nabla}\langle\mathrm{c}\rangle^{\alpha}\right\}+\mathrm{z}_{+} \underline{\nabla} \bullet\left\{\mathrm{a}_{\mathrm{v}} \underline{\underline{D}}_{\mathrm{eff}}^{\mathrm{m}} \bullet\langle\mathrm{q}\rangle_{\alpha \beta} \underline{\nabla}\langle\psi\rangle^{\alpha}\right\}-\mathrm{a}_{\mathrm{v}} \frac{\partial \mathrm{w}\rangle_{\alpha \beta}}{\partial \mathrm{t}} .
$$

Here $\underline{\underline{D}}^{*}$ is a global effective diffusivity tensor given by:

$$
\underline{\underline{D}}^{*}=\underline{\underline{D}}_{\text {eff }}+\underline{\underline{D}}_{\mathrm{eff}}^{\mathrm{s}} \frac{\mathrm{a}_{\mathrm{v}}}{\varepsilon_{\alpha}} \frac{\langle\mathrm{w}\rangle_{\alpha \beta}}{\langle\mathrm{c}\rangle^{\alpha}}
$$

Ochoa-Tapia et al. (1993) used a linear isotherm to relate surface $\left(\mathrm{c}_{\mathrm{is}}\right)$ and bulk $\left(\mathrm{c}_{\mathrm{i}}\right)$ salt concentrations; therefore, the effective diffusivity tensors calculated using equations similar to (45) are independent of bulk and surface salt concentrations. In charged porous media, Eq. (46) shows that the transport parameters are functions of the ratio $\left(\langle\mathrm{w}\rangle_{\alpha \beta} /\langle\mathrm{c}\rangle^{\alpha}\right)$ that varies in a highly nonlinear way throughout the porous electrode. According to the GCS model, we can derive the following expression:

$$
\frac{\langle\mathrm{w}\rangle_{\alpha \beta}}{\langle\mathrm{c}\rangle^{\alpha}}=8 \frac{\lambda_{\mathrm{D}}}{\sqrt{\langle\mathrm{c}\rangle^{\alpha} / \mathrm{C}_{\infty}}} \sinh ^{2}\left(\Delta \psi_{\mathrm{D}} / 4\right)
$$

Here, $\Delta \psi_{\mathrm{D}}$ is the dimensionless potential drop in the diffuse layer. Eq. (47) shows that the effective diffusivities will vary as $\langle\mathrm{c}\rangle^{\alpha}$ and $\Delta \psi_{\mathrm{D}}$ vary inside the porous electrode. This issue will be discussed in the results section.

\subsection{Electrical Potential}


We start our derivation from Eq. (7) and the boundary condition at the $\alpha-\beta$ interphase given by Eq. (12). Again, the boundary conditions in the input-output area of the macroscopic domain are not relevant to the volume averaging method, as the problem can be expressed by a local problem. The physical system defined by Eqs. (7) and (12) is somewhat similar to the diffusiondispersion problem with heterogeneous reaction and/or absorption studied by several authors (Carbonell and Whitaker, 1983; Quintard and Whitaker, 1998; Ochoa-Tapia et al., 1994; Borges da Silva et al., 2001; Ulson de Souza and Whitaker, 2003). In the dispersion studies, we have an independently calculated vector field $(\underline{v})$ that influences the value of the concentration scalar field. In this work, however, the concentration and potential gradient distributions cannot be calculated independently. We proceed applying the phase average operation defined by eq. (13) to both sides of eq. (7). Repeated use of the tools described above plus the assumptions: $\mathrm{w} \cong\langle\mathrm{w}\rangle_{\alpha \beta}, \mathrm{q} / \mathrm{c} \cong\langle\mathrm{q}\rangle_{\alpha \beta} /\langle\mathrm{c}\rangle^{\alpha}$ leads to:

$$
\begin{aligned}
& \underline{\nabla} \bullet\left\{\varepsilon_{\alpha} \mathrm{D}\langle\mathrm{c}\rangle^{\alpha}\left[\underline{\nabla}\langle\psi\rangle^{\alpha}+\frac{1}{\mathrm{~V}_{\alpha}} \int_{\mathrm{A}_{\alpha \beta}} \underline{\mathrm{n}}_{\alpha \beta} \tilde{\psi} \mathrm{dA}\right]\right\}+\underline{\nabla} \bullet\left\{\mathrm{a}_{\mathrm{v}} \mathrm{D}_{\mathrm{s}} \frac{\langle\mathrm{q}\rangle_{\alpha \beta}}{\langle\mathrm{c}\rangle^{\alpha}}\left[\underline{\nabla}\langle\mathrm{c}\rangle^{\alpha}+\frac{1}{\mathrm{~A}_{\alpha \beta}} \int_{\mathrm{A}_{\alpha \beta}} 2 \mathrm{H}_{\alpha \beta} \tilde{\mathrm{c}} \mathrm{dA}\right]\right\} \\
& +\mathrm{z}_{+} \underline{\nabla} \bullet\left\{\mathrm{a}_{\mathrm{v}} \mathrm{D}_{\mathrm{s}}\langle\mathrm{w}\rangle_{\alpha \beta}\left[\underline{\nabla}\langle\psi\rangle^{\alpha}+\frac{1}{\mathrm{~A}_{\alpha \beta}} \int_{\mathrm{A}_{\alpha \beta}} 2 \mathrm{H} \underline{\mathrm{n}}_{\alpha \beta} \tilde{\psi} \mathrm{dA}\right]\right\}-\mathrm{a}_{\mathrm{v}} \frac{\partial \mathrm{q}\rangle_{\alpha \beta}}{\partial \mathrm{t}}=0
\end{aligned}
$$

We have already developed an expression for the salt concentration deviations appearing in Eq. (47). However, we also need a similar expression for the electrical potential deviations ( $\tilde{\psi}$ ) in order to close Eq. (47). This task will be accomplished in next section.

\subsubsection{Closure for Electrical Potential Equation}

We start our derivation from the boundary condition at the $\alpha-\beta$ interface given by Eq. (12). After algebraic manipulations we obtain:

$$
\begin{aligned}
& \underline{\mathrm{n}}_{\alpha \beta} \bullet(\mathrm{D} \mathrm{c} \underline{\nabla} \tilde{\psi})-\mathrm{z}_{+} \underline{\nabla}_{\mathrm{s}} \bullet\left[\mathrm{D}_{\mathrm{s}}\langle\mathrm{w}\rangle_{\alpha \beta} \underline{\nabla}_{\mathrm{s}} \tilde{\psi}\right]=\underline{\nabla}_{\mathrm{s}} \bullet\left[\mathrm{D}_{\mathrm{s}} \frac{\langle\mathrm{q}\rangle_{\alpha \beta}}{\langle\mathrm{c}\rangle^{\alpha}} \underline{\nabla}_{\mathrm{s}} \mathrm{c}\right]+\mathrm{z}_{+} \underline{\nabla}_{\mathrm{s}} \bullet\left[\mathrm{D}_{\mathrm{s}}\langle\mathrm{w}\rangle_{\alpha \beta} \underline{\nabla}_{\mathrm{s}}\langle\psi\rangle^{\alpha}\right] \\
& -\underline{\mathrm{n}}_{\alpha \beta} \bullet\left(\mathrm{D} \mathrm{c} \underline{\nabla}\langle\psi\rangle^{\alpha}\right)-\frac{\partial\langle\mathrm{q}\rangle_{\alpha \beta}}{\partial \mathrm{t}} .
\end{aligned}
$$

After introduction of Eq. (27) into Eq. (48) and algebraic manipulations we get a much simplified problem given by:

$$
\begin{aligned}
& \underline{\mathrm{n}}_{\alpha \beta} \bullet(\mathrm{D} \mathrm{c} \underline{\nabla} \tilde{\psi})-\mathrm{z}_{+} \underline{\nabla}_{\mathrm{s}} \bullet\left[\mathrm{D}_{\mathrm{s}}\langle\mathrm{w}\rangle_{\alpha \beta} \underline{\nabla}_{\mathrm{s}} \tilde{\psi}\right]=-2 \mathrm{H} \underline{\underline{n}}_{\alpha \beta} \bullet\left[\mathrm{D}_{\mathrm{s}} \frac{\langle\mathrm{q}\rangle_{\alpha \beta}}{\langle\mathrm{c}\rangle^{\alpha}} \underline{\nabla} \mathrm{c}\right] \\
& -\underline{\mathrm{n}}_{\alpha \beta} \bullet\left[\mathrm{Dc}+\mathrm{z}_{+} 2 \mathrm{H} \mathrm{D}_{\mathrm{s}}\langle\mathrm{w}\rangle_{\alpha \beta}\right] \underline{\nabla}\langle\psi\rangle^{\alpha}-\frac{\partial\langle\mathrm{q}\rangle_{\alpha \beta}}{\partial \mathrm{t}} .
\end{aligned}
$$

After inspection of Eq. (49), we propose that:

$$
\left.\widetilde{\psi}=\underline{\mathrm{g}}_{1} \bullet \underline{\nabla}\langle\mathrm{c}\rangle^{\alpha}+\underline{\mathrm{g}}_{2} \bullet \underline{\nabla}\langle\psi\rangle^{\alpha}+\mathrm{g}_{3} \partial \mathrm{q}\right\rangle_{\alpha \beta} / \partial \mathrm{t}
$$

We obtain the equation for the dimensionless potential deviation $(\tilde{\psi})$ by subtracting Eq. (47) divided by $\varepsilon_{\alpha}$ from Eq. (7) to obtain: 


$$
\left.\underline{\nabla} \bullet(\mathrm{D} \mathrm{c} \underline{\nabla} \tilde{\psi})=\underline{\nabla} \bullet\left[\mathrm{D} \tilde{\mathrm{c}}+\frac{\mathrm{a}_{\mathrm{v}}}{\varepsilon_{\alpha}} \mathrm{D}_{\mathrm{s}}\langle\mathrm{q}\rangle_{\alpha \beta}\right] \underline{\nabla}\langle\psi\rangle^{\alpha}\right)+\underline{\nabla} \bullet\left(\frac{\mathrm{a}_{\mathrm{v}}}{\varepsilon_{\alpha}} \mathrm{D}_{\mathrm{s}} \frac{\langle\mathrm{w}\rangle_{\alpha \beta}}{\langle\mathrm{c}\rangle^{\alpha}} \underline{\nabla}\langle\mathrm{c}\rangle^{\alpha}\right)-\frac{\mathrm{a}_{\mathrm{v}}}{\varepsilon_{\alpha}} \frac{\partial \mathrm{w}\rangle_{\alpha \beta}}{\partial \mathrm{t}} .
$$

An order of magnitude analysis shows that the integral terms are negligible compared to the first on the right-hand-side of Eq. (51). The second and third terms are also negligible due to the constraints given in Eq. (27). In conclusion, the equation to calculate the potential deviations is reduced to:

$$
\underline{\nabla} \bullet(\mathrm{Dc} \underline{\nabla} \tilde{\psi})=-\frac{\mathrm{a}_{\mathrm{v}}}{\varepsilon_{\alpha}} \frac{\partial \mathrm{q}\rangle_{\alpha \beta}}{\partial \mathrm{t}} .
$$

The $\underline{g}_{1}$ and $\underline{g}_{2}$ vector fields, and the scalar field $g_{3}$ are calculated by solving the following boundary-value problems:

Problem 4

$$
\underline{\nabla}^{2} \underline{\mathrm{g}}_{1}=0
$$

$\underline{\mathrm{n}}_{\alpha \beta} \bullet\left(\mathrm{D}\langle\mathrm{c}\rangle^{\alpha} \underline{\nabla}_{\mathrm{g}_{1}}\right)-\mathrm{z}_{+} \underline{\nabla}_{\mathrm{s}} \bullet\left[\mathrm{D}_{\mathrm{s}}\langle\mathrm{w}\rangle_{\alpha \beta} \underline{\nabla}_{\mathrm{s}} \underline{\mathrm{g}}_{1}\right]=-2 \mathrm{H} \underline{\mathrm{n}}_{\alpha \beta} \bullet\left[\mathrm{D}_{\mathrm{s}}\langle\mathrm{q}\rangle_{\alpha \beta}\right]$,

at the $A_{\alpha \beta}$ and for spatially periodic porous media:

$$
\underline{\mathrm{g}}_{1}\left(\underline{\mathrm{r}}+\underline{\mathrm{l}}_{\mathrm{i}}\right)=\underline{\mathrm{g}}_{1}(\underline{\mathrm{r}})
$$

Problem 5

$$
\underline{\nabla}^{2} \underline{\mathrm{g}}_{2}=0
$$

$$
\underline{\mathrm{n}}_{\alpha \beta} \bullet\left(\underline{\nabla}_{2}\right)-\mathrm{z}_{+} \underline{\nabla}_{\mathrm{s}} \bullet\left[\frac{\mathrm{D}_{\mathrm{s}}\langle\mathrm{w}\rangle_{\alpha \beta}}{\mathrm{D}\langle\mathrm{c}\rangle^{\alpha}} \underline{\nabla}_{\mathrm{s}} \underline{\mathrm{g}}_{2}\right]=-\underline{\mathrm{n}}_{\alpha \beta}\left[1+\mathrm{z}_{+} 2 \mathrm{H} \frac{\mathrm{D}_{\mathrm{s}}\langle\mathrm{w}\rangle_{\alpha \beta}}{\mathrm{D}\langle\mathrm{c}\rangle^{\alpha}}\right],
$$

at the $\mathrm{A}_{\alpha \beta}$ and for spatially periodic porous media:

$\underline{\mathrm{g}}_{2}\left(\underline{\mathrm{r}}+\underline{\mathrm{l}}_{\mathrm{i}}\right)=\underline{\mathrm{g}}_{2}(\underline{\mathrm{r}})$.

Problem 6

$$
\begin{aligned}
& \underline{\nabla}^{2} \mathrm{~g}_{3}=-\mathrm{a}_{\mathrm{v}} /\left(\varepsilon_{\alpha} \mathrm{D}\langle\mathrm{c}\rangle^{\alpha}\right), \\
& \underline{\mathrm{n}}_{\alpha \beta} \underline{\nabla}_{3}-\mathrm{z}_{+} \frac{\mathrm{D}_{\mathrm{s}}}{\mathrm{D}} \frac{\langle\mathrm{w}\rangle_{\alpha \beta}}{\langle\mathrm{c}\rangle^{\alpha}} \nabla_{\mathrm{s}}^{2} \mathrm{~g}_{3}=-\frac{1}{\mathrm{D}\langle\mathrm{c}\rangle^{\alpha}},
\end{aligned}
$$

at the $\mathrm{A}_{\alpha \beta}$ and also for spatially periodic porous media.

$\underline{\mathrm{g}}_{3}\left(\underline{\mathrm{r}}+\underline{\mathrm{l}}_{\mathrm{i}}\right)=\underline{\mathrm{g}}_{3}(\underline{\mathrm{r}})$.

Inspection of the closure problems shows that, if $\mathrm{z}_{+}=1$, problem 4 is identical to problem 2 .

\subsubsection{Closed Electrical Potential Equation}

We start our derivation by introducing the derived expressions for salt concentration deviations, Eq. (29), and electrical potential deviations, Eq. (50), into Eq. (47). After complex algebraic manipulations we obtain: 


$$
\begin{aligned}
& \underline{\nabla} \bullet\left\{\varepsilon_{\alpha} \underline{\underline{U}}_{\text {eff }}\langle\mathrm{c}\rangle^{\alpha} \bullet \underline{\nabla}\langle\psi\rangle^{\alpha}\right\}+\underline{\nabla} \bullet\left\{\mathrm{a}_{\mathrm{v}} \underline{\underline{U}}_{\text {eff }}^{\mathrm{s}} \frac{\langle\mathrm{q}\rangle_{\alpha \beta}}{\langle\mathrm{c}\rangle^{\alpha}} \bullet \underline{\nabla}\langle\mathrm{c}\rangle^{\alpha}\right\} \\
& +\mathrm{z}_{+} \underline{\nabla} \bullet\left\{\mathrm{a}_{\mathrm{v}} \underline{\underline{\mathrm{U}}}_{\text {eff }}^{\mathrm{m}}\langle\mathrm{w}\rangle_{\alpha \beta} \bullet \underline{\nabla}\langle\psi\rangle^{\alpha}\right\}-\mathrm{a}_{\mathrm{v}} \frac{\partial \mathrm{q}\rangle_{\alpha \beta}}{\partial \mathrm{t}}=0
\end{aligned}
$$

Once again the nontraditional terms have been eliminated using the constraints given by Eq. (27). In Eq. (62), the first term on the left-hand side represents bulk migration while the second and third represent surface transport processes. The last term on the left-hand side represents charge accumulation in the EDLs. The transport tensors appearing in Eq. (62) are given by:

$$
\begin{aligned}
& \underline{\mathrm{U}}_{\mathrm{eff}}=\mathrm{D}\left[\underline{\underline{\mathrm{I}}}+\frac{1}{\mathrm{~V}_{\alpha}} \int_{\mathrm{A}_{\alpha \beta}} \underline{\mathrm{n}}_{\alpha \beta} \underline{\mathrm{g}}_{2} \mathrm{dA}\right] \\
& \underline{\underline{\mathrm{U}}}_{\mathrm{eff}}^{\mathrm{s}}=\mathrm{D}_{\mathrm{s}}\left\{\left[\underline{\underline{\mathrm{I}}}+\frac{1}{\mathrm{~A}_{\alpha \beta}} \int_{\mathrm{A}_{\alpha \beta}} 2 \mathrm{H} \underline{\underline{n}}_{\alpha \beta} \underline{\mathrm{f}}_{1} \mathrm{dA}+\mathrm{z}_{+} \frac{\langle\mathrm{c}\rangle^{\alpha}\langle\mathrm{w}\rangle_{\alpha \beta}}{\langle\mathrm{q}\rangle_{\alpha \beta}} \frac{1}{\mathrm{~A}_{\alpha \beta}} \int_{\mathrm{A}_{\alpha \beta}} 2 \mathrm{H}_{\underline{n}_{\alpha \beta}} \underline{\mathrm{g}}_{1} \mathrm{dA}\right.\right. \\
& \left.+\frac{\mathrm{Z}_{+} \mathrm{D}\langle\mathrm{c}\rangle^{\alpha}}{\mathrm{D}_{\mathrm{s}}\langle\mathrm{q}\rangle_{\alpha \beta} 2 \mathrm{H}} \frac{\langle\mathrm{c}\rangle^{\alpha} 2 \mathrm{H}}{\mathrm{A}_{\alpha \beta}} \int_{\mathrm{A}_{\alpha \beta}} \underline{\underline{n}}_{\alpha \beta} \underline{\mathrm{g}}_{1} \mathrm{dA}\right] \\
& \underline{\underline{\mathrm{U}}}_{\mathrm{eff}}^{\mathrm{m}}=\mathrm{D}_{\mathrm{s}}\left\{\underline{\underline{\mathrm{I}}}+\frac{1}{\mathrm{~A}_{\alpha \beta}} \int_{\mathrm{A}_{\alpha \beta}} 2 \mathrm{H} \underline{\mathrm{n}}_{\alpha \beta} \underline{\mathrm{g}}_{2} \mathrm{dA}+\frac{\langle\mathrm{q}\rangle_{\alpha \beta}}{\langle\mathrm{c}\rangle^{\alpha}\langle\mathrm{w}\rangle_{\alpha \beta}} \frac{1}{\mathrm{~A}_{\alpha \beta}} \int_{\mathrm{A}_{\alpha \beta}} 2 \mathrm{H} \underline{\mathrm{n}}_{\alpha \beta} \mathrm{f}_{2} \mathrm{dA}\right\} .
\end{aligned}
$$

In the calculation of transport parameters, it is important to point out that, if $\mathrm{z}_{+}$is equal to one, problems 1 and 5 are identical; therefore, the tensor $\underline{\underline{U}}_{\text {eff }}$ is equal to the tensor $\underline{\underline{D}}_{\text {eff }}$. In order to calculate the tensors, $\left(\underline{\underline{U}}_{\text {eff }}^{\mathrm{s}}\right)$ and $\left(\underline{\underline{U}}_{\text {eff }}^{\mathrm{m}}\right)$ we also need to solve the closure problem 4 .

If the surface transport terms are negligible compared to bulk migration, we obtain the following equation:

$$
\underline{\nabla} \bullet\left\{\varepsilon_{\alpha} \underline{\underline{U}}_{\text {eff }} \bullet\langle\mathrm{c}\rangle^{\alpha} \underline{\nabla}\langle\psi\rangle^{\alpha}\right\}-\mathrm{a}_{\mathrm{v}} \partial\langle\mathrm{q}\rangle_{\alpha \beta} / \partial \mathrm{t}=0 .
$$

Eq. (64) is identical to the expression proposed by Biesheuvel and Bazant (2010) and the equivalent one derived by Gabitto and Tsouris (2015). We can combine bulk and surface migration into a single global term. After some algebraic manipulations of Eq. (62) we obtain:

$$
\underline{\nabla} \bullet\left\{\varepsilon_{\alpha} \underline{\underline{U}}^{*} \bullet\langle\mathrm{c}\rangle^{\alpha} \underline{\nabla}\langle\psi\rangle^{\alpha}\right\}+\underline{\nabla} \bullet\left\{\mathrm{a}_{\mathrm{v}} \underline{\underline{U}}_{\text {eff }}^{\mathrm{s}} \bullet \frac{\langle\mathrm{q}\rangle_{\alpha \beta}}{\langle\mathrm{c}\rangle^{\alpha}} \underline{\nabla}\langle\mathrm{c}\rangle^{\alpha}\right\}-\mathrm{a}_{\mathrm{v}} \frac{\partial \mathrm{q}\rangle_{\alpha \beta}}{\partial \mathrm{t}}=0 .
$$

Here, $\underline{\underline{U}}^{*}$ is the global effective mobility tensor defined by:

$$
\underline{\underline{U}}^{*}=\underline{\underline{U}}_{\text {eff }}+\mathrm{U}_{\text {eff }}^{\mathrm{m}} \frac{\mathrm{a}_{\mathrm{v}}}{\varepsilon_{\alpha}} \frac{\langle\mathrm{w}\rangle_{\alpha \beta}}{\langle\mathrm{c}\rangle^{\alpha}} .
$$

Eq. (65) shows that all surface transport processes can be described by a single surface migration-like term. This statement means that the influence of surface diffusion is lumped together with bulk diffusion in a combined term controlled by a global effective mobility. However, the global effective mobility is not a constant dependent only on geometrical factors. It also depends on the $\langle w\rangle_{\alpha \beta} /\langle\mathrm{c}\rangle^{\alpha}$ ratio which varies in a highly nonlinear way.

\section{Closure Problem Solution}




\subsection{Problem Derivation}

In order to estimate the value of the transport properties, we will follow the approach reported by Prof. S. Whitaker and co-workers who solved the closure problems 1, 2, 4, and 5 in isotropic spatially periodic unit cells. Figure 2 depicts a spatially periodic porous medium where the particles are either cylinders of infinite length or spheres. This system is represented by a square unit cell containing a circular particle in the case of infinite length cylinders or a cubic cell containing a body centered spherical particle. Kuwabara (1959) reported for the first time the use of cell models to study transport phenomena in isotropic systems. Chang (1982 and 1983) proved that in the approximate (circular) unit cell shown in Figure 2 the periodic boundary condition on the cell boundary $\left(r=r_{2}\right)$ can be replaced by a zero Dirichlet boundary condition. The use of the Dirichlet boundary condition leads to the existence of an analytical solution. Ochoa (1988) examined in detail numerically the issue and concluded that reasonably good agreement exists between the numerical solutions in the real unit cell and the analytical ones in Chang's unit cell depicted in Figure 2.

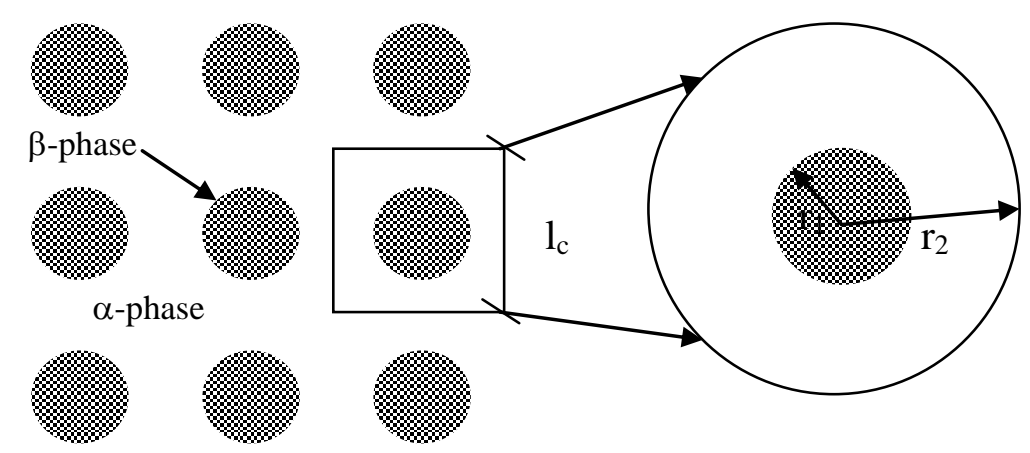

Figure 2. Schematic of the isotropic spatially periodic porous medium including Chang's unit cell.

It is obvious that the unit cell shown in Figure 2 will lead to an isotropic effective diffusivity tensor; thus, we need only to determine one $\underline{f}_{1}$ component $\left(f_{x}\right)$ in order to predict the transport properties. Under these circumstances, the closure problem 1 is given by:

$$
\begin{aligned}
& \underline{\nabla}^{2} \mathrm{f}_{\mathrm{x}}=0 \\
& \underline{\mathrm{n}}_{\alpha \beta} \bullet \underline{\nabla f}_{\mathrm{x}}-\left[\frac{\mathrm{D}_{\mathrm{s}}}{\mathrm{D}} \frac{\langle\mathrm{w}\rangle_{\alpha \beta}}{\langle\mathrm{c}\rangle^{\alpha}} \nabla_{\mathrm{s}}^{2} \mathrm{f}_{\mathrm{x}}\right]=-\left(\underline{\mathrm{i}} \bullet \underline{\mathrm{n}}_{\alpha \beta}\right)\left[2 \mathrm{H} \frac{\mathrm{D}_{\mathrm{s}}}{\mathrm{D}} \frac{\langle\mathrm{w}\rangle_{\alpha \beta}}{\langle\mathrm{c}\rangle^{\alpha}}+1\right],
\end{aligned}
$$

at the $\mathrm{A}_{\alpha \beta}$ interface

$$
\mathrm{f}_{\mathrm{x}}=0 \text {, at } \mathrm{r}=\mathrm{r}_{2}
$$

Here, $\mathrm{f}_{\mathrm{x}}$ is given by $\underline{\mathrm{n}}_{\alpha \beta} \bullet \underline{\mathrm{f}}_{1}$.

\subsection{Cylindrical Unit Cell}

\subsubsection{Species Continuity Equation}

In this case the problem becomes independent of the axial direction $\left(\mathrm{z} / \mathrm{r}_{2}\right)$ and, following the useful relationships presented by Ochoa-Tapia et al. (1993), we can rewrite the closure problem as, 


$$
\begin{aligned}
& \frac{1}{\mathrm{r}^{*}} \frac{\partial \mathrm{F}_{\mathrm{x}}}{\partial \mathrm{r}^{*}}+\frac{\partial^{2} \mathrm{~F}_{\mathrm{x}}}{\partial \mathrm{r}^{* 2}}+\frac{1}{\mathrm{r}^{* 2}} \frac{\partial^{2} \mathrm{~F}_{\mathrm{x}}}{\partial \theta^{2}}=0 \\
& -\frac{\partial \mathrm{F}_{\mathrm{x}}}{\partial \mathrm{r}^{*}}-\left(\frac{\mathrm{r}_{2}}{\mathrm{r}_{1}}\right) \Lambda_{\mathrm{w}} \frac{\partial^{2} \mathrm{~F}_{\mathrm{x}}}{\partial \theta^{2}}=\left(1-\Lambda_{\mathrm{w}}\right) \cos \theta, \text { at } \mathrm{r}^{*}=\mathrm{r}_{1} / \mathrm{r}_{2} \\
& \mathrm{~F}_{\mathrm{x}}=0, \text { at } \mathrm{r}^{*}=1
\end{aligned}
$$

Here, $\Lambda_{\mathrm{w}}=2 \xi \frac{\mathrm{D}_{\mathrm{s}}}{\mathrm{D}} \frac{\left\langle\mathrm{w}^{*}\right\rangle_{\alpha \beta}}{\left\langle\mathrm{c}^{*}\right\rangle^{\alpha}}$ and $\xi=\lambda_{\mathrm{D}} / \mathrm{r}_{1}$. This closure problem is identical to the one presented by Ochoa-Tapia et al. (1993); however, the definition of the parameter $\Lambda_{w}$ is different. The solution procedure detailed in the supplementary information (Appendix A) leads to:

$$
\frac{\varepsilon_{\alpha} \mathrm{D}_{\mathrm{xx}}}{\mathrm{D}}=\frac{\varepsilon_{\alpha}\left[\left(1+\Lambda_{\mathrm{w}}\right)\right]}{\left(2-\varepsilon_{\alpha}\right)+\varepsilon_{\alpha} \Lambda_{\mathrm{w}}}
$$

If $\Lambda_{\mathrm{w}} \ll<1$, we obtain Rayleigh's (1892) solution for the bulk effective diffusivity:

$$
\frac{\varepsilon_{\alpha} \mathrm{D}_{\mathrm{xx}}^{\mathrm{b}}}{\mathrm{D}}=\frac{\varepsilon_{\alpha}}{\left(2-\varepsilon_{\alpha}\right)}
$$

Inspection of Eq. (73) shows that the effective diffusivity calculated solving problem 1 is not a true bulk diffusivity coefficient because depends upon the geometry and the value of the parameter $\Lambda_{\mathrm{w}}$, which depends upon the surface transport process. Bulk effective diffusivities are assumed to depend only upon the geometric parameters of the porous media (Whitaker, 1999). This is the main difference with the bulk effective diffusivity calculated by Gabitto and Tsouris (2015), and many other authors, for similar transport processes. Ochoa-Tapia et al. (1993) encountered similar problem in their study of surface transport in uncharged porous media. The reason is the mathematical structure of the closure problem 1 . The solution is generated by the boundary condition given by Eq. (71). In the surface transport case, there are two contributions to the closure parameter field $\underline{\mathrm{f}}_{1}$, one given by the bulk process and another given by the surface transport process. Therefore, we propose to split the transport coefficient given by Eq. (73) into:

$$
\frac{\varepsilon_{\alpha} \mathrm{D}_{\mathrm{xx}}}{\mathrm{D}}=\frac{\varepsilon_{\alpha} \mathrm{D}_{\mathrm{xx}}^{\mathrm{b}}}{\mathrm{D}}+\frac{\varepsilon_{\alpha} \mathrm{D}_{\mathrm{xx}}^{\mathrm{bs}}}{\mathrm{D}}
$$

Here, $\frac{\varepsilon_{\alpha} D_{x x}^{b s}}{D}$ is the surface contribution to the effective diffusivity. Using Eq. (75), we can algebraically calculate the value of the surface contribution as:

$$
\frac{\varepsilon_{\alpha} \mathrm{D}_{\mathrm{xx}}^{\mathrm{bs}}}{\mathrm{D}}=\frac{2 \varepsilon_{\alpha} \Lambda_{\mathrm{w}}\left(1-\varepsilon_{\alpha}\right)}{\left[\left(2-\varepsilon_{\alpha}\right)+\varepsilon_{\alpha} \Lambda_{\mathrm{w}}\right]\left(2-\varepsilon_{\alpha}\right)} .
$$

In the case of $\mathrm{z}_{+}=1$, simple inspection shows that problems 1 and 5 are identical; therefore, the closure parameter fields $\underline{f}_{1}$ and $\underline{g}_{2}$ are identical. Thus, the bulk mobility tensor is also identical to the bulk diffusivity tensor. The calculation of the other four mass transport coefficients also requires solution of the closure problems 2 and 4 . However, both are identical when $\mathrm{z}_{+}=1$. In the supplementary information (Appendix B), we show the solution to both closure problems. In 
the solution of these closure problems, we defined the parameter $\Lambda_{q}=2 \xi \frac{D_{s}}{D} \frac{\left\langle q^{*}\right\rangle_{\alpha \beta}}{\left\langle c^{*}\right\rangle^{\alpha}}$. This parameter represents the influence of the excess charge density on the surface transport process. At the same time, the ratio $\Lambda_{\mathrm{w}} / \Lambda_{\mathrm{q}}$ represents a key property of porous electrodes, the charge efficiency of the double layer defined as the ratio of excess salt adsorption over excess electrode charge: $\left\langle\mathrm{w}^{*}\right\rangle_{\alpha \beta} /\left\langle\mathrm{q}^{*}\right\rangle_{\alpha \beta}$ according to Zhao et al. (2010). After application of the solution procedure shown in the supplementary information (Appendix B), we found that:

$$
\begin{aligned}
& \frac{\mathrm{D}_{\mathrm{xx}}^{\mathrm{s}}}{\mathrm{D}_{\mathrm{s}}}=\frac{\left[4-\varepsilon_{\alpha}+\varepsilon_{\alpha} \Lambda_{\mathrm{w}}\right] \Lambda_{\mathrm{w}}-\mathrm{z}_{+} \varepsilon_{\alpha} \Lambda_{\mathrm{q}}^{2}}{2\left[\left(2-\varepsilon_{\alpha}\right)+\varepsilon_{\alpha} \Lambda_{\mathrm{w}}\right] \Lambda_{\mathrm{w}}}, \\
& \frac{\mathrm{D}_{\mathrm{xx}}^{\mathrm{m} *}}{\mathrm{D}}=\frac{\mathrm{D}_{\mathrm{xx}}^{\mathrm{m}}}{\mathrm{D}_{\mathrm{s}}} \frac{\mathrm{a}_{\mathrm{v}}\langle\mathrm{q}\rangle_{\alpha \beta}}{\langle\mathrm{c}\rangle^{\alpha}} \frac{\mathrm{D}_{\mathrm{s}}}{\mathrm{D}}=\frac{\mathrm{D}_{\mathrm{xx}}^{\mathrm{m}}}{\mathrm{D}_{\mathrm{s}}} 2 \Lambda_{\mathrm{q}}\left(1-\varepsilon_{\alpha}\right)=\frac{4 \Lambda_{\mathrm{q}}\left(1-\varepsilon_{\alpha}\right)}{\left[\left(2-\varepsilon_{\beta}\right)+\varepsilon_{\beta} \Lambda_{\mathrm{w}}\right]} .
\end{aligned}
$$

Eqns. (77) and (78) allow calculation of the value of the effective surface diffusivity and the value of the effective surface migration transport coefficient, respectively. However, as we discussed above there is also a contribution to the surface diffusivity coming from the bulk coefficient $\mathrm{D}_{\mathrm{xx}}^{\mathrm{bs}}$. Therefore, we can collect all the contributions to surface diffusion in a single surface diffusion transport coefficient $\left(\mathrm{D}_{\mathrm{xx}}^{\mathrm{s*}}\right)$ given by,

$$
\begin{aligned}
\frac{\mathrm{D}_{\mathrm{xx}}^{\mathrm{s*}}}{\mathrm{D}} & =\frac{\varepsilon_{\alpha} \mathrm{D}_{\text {eff }}^{\mathrm{bs}}}{\mathrm{D}}+\frac{\mathrm{D}_{\mathrm{xx}}^{\mathrm{s}}}{\mathrm{D}_{\mathrm{s}}} 2 \Lambda_{\mathrm{w}}\left(1-\varepsilon_{\alpha}\right) \\
\frac{\mathrm{D}_{\mathrm{xx}}^{\mathrm{s*}}}{\mathrm{D}} & =\frac{2 \varepsilon_{\alpha} \Lambda_{\mathrm{w}}\left(1-\varepsilon_{\alpha}\right)+\left[\left(4-\varepsilon_{\alpha}+\varepsilon_{\alpha} \Lambda_{\mathrm{w}}\right) \Lambda_{\mathrm{w}}-\mathrm{z}_{+} \varepsilon_{\beta} \Lambda_{\mathrm{q}}^{2}\right]\left(1-\varepsilon_{\alpha}\right)\left(2-\varepsilon_{\beta}\right)}{\left[\left(2-\varepsilon_{\beta}\right)+\varepsilon_{\beta} \Lambda_{\mathrm{w}}\right]\left(2-\varepsilon_{\beta}\right)} .
\end{aligned}
$$

The arrangement of terms shown in Eq. (79) leads to the following closed equation for the species continuity balance:

$$
\varepsilon_{\alpha} \frac{\partial \mathrm{c}\rangle^{\alpha}}{\partial \mathrm{t}}=\underline{\nabla} \bullet\left[\varepsilon_{\alpha} \underline{\underline{D}}_{\mathrm{eff}}^{\mathrm{b}}+\underline{\underline{D}}_{\mathrm{eff}}^{\mathrm{s}^{*}}\right] \bullet \underline{\nabla}\langle\mathrm{c}\rangle^{\alpha}+\mathrm{z}_{+} \underline{\nabla} \bullet \underline{\underline{D}}_{\mathrm{eff}}^{\mathrm{m}^{*}} \bullet\langle\mathrm{c}\rangle^{\alpha} \underline{\nabla}\langle\psi\rangle^{\alpha}-\mathrm{a}_{\mathrm{v}} \frac{\partial \mathrm{w}\rangle_{\alpha \beta}}{\partial \mathrm{t}} .
$$

In Eq. (81), the bulk effective diffusivity $\left(\underline{\underline{D}}_{\text {eff }}^{\mathrm{b}}\right)$ depends only upon the geometry of the porous medium, and all the contributions from surface diffusion terms have been collected into the second term on the right-hand side. We can also follow Ochoa-Tapia et al. (1993) in combining the surface and bulk diffusion terms into a global diffusion term using:

$$
\frac{\varepsilon_{\alpha} \mathrm{D}_{\mathrm{xx}}^{*}}{\mathrm{D}}=\frac{\varepsilon_{\alpha} \mathrm{D}_{\mathrm{xx}}^{\mathrm{b}}}{\mathrm{D}}+\frac{\mathrm{D}_{\mathrm{xx}}^{\mathrm{s}^{*}}}{\mathrm{D}}
$$

Eq. (82) leads to:

$$
\frac{\mathrm{D}_{\mathrm{xx}}^{*}}{\mathrm{D}}=\frac{\varepsilon_{\alpha}\left[\left(2-\varepsilon_{\alpha}\right)+\varepsilon_{\alpha} \Lambda_{\mathrm{w}}\right]+\left[\left(4-\varepsilon_{\alpha}+\varepsilon_{\alpha} \Lambda_{\mathrm{w}}\right) \Lambda_{\mathrm{w}}-\mathrm{z}_{+} \varepsilon_{\beta} \Lambda_{\mathrm{q}}^{2}\right]\left(1-\varepsilon_{\alpha}\right)\left(2-\varepsilon_{\alpha}\right)}{\left[\left(2-\varepsilon_{\alpha}\right)+\varepsilon_{\alpha} \Lambda_{\mathrm{w}}\right]\left(2-\varepsilon_{\alpha}\right)} .
$$

In this case, the salt concentration equation is given by:

$$
\varepsilon_{\alpha} \frac{\partial \mathrm{c}\rangle^{\alpha}}{\partial \mathrm{t}}=\underline{\nabla} \bullet\left\{\varepsilon_{\alpha} \underline{\underline{D}}_{\mathrm{eff}}^{*} \bullet \underline{\nabla}\langle\mathrm{c}\rangle^{\alpha}\right\}+\mathrm{z}_{+} \underline{\nabla} \bullet\left\{\underline{\underline{\mathrm{D}}}_{\mathrm{eff}}^{\mathrm{m}^{*}}\langle\mathrm{c}\rangle^{\alpha} \bullet \underline{\nabla}\langle\psi\rangle^{\alpha}\right\}-\mathrm{a}_{\mathrm{v}} \frac{\partial \mathrm{w}\rangle_{\alpha \beta}}{\partial \mathrm{t}} .
$$

\subsubsection{Electrical Potential}


The fact that closure problems 1 and 5 are identical leads to identical equations for the effective mobility transport coefficient:

$$
\frac{\varepsilon_{\alpha} \mathrm{D}_{\mathrm{xx}}^{\mathrm{bs}}}{\mathrm{D}}=\frac{\varepsilon_{\alpha} \mathrm{U}_{\mathrm{xx}}^{\mathrm{bs}}}{\mathrm{D}}=\frac{2 \varepsilon_{\alpha} \Lambda_{\mathrm{w}}\left(1-\varepsilon_{\alpha}\right)}{\left[\left(2-\varepsilon_{\alpha}\right)+\varepsilon_{\alpha} \Lambda_{\mathrm{w}}\right]\left(2-\varepsilon_{\alpha}\right)} .
$$

The solution of the closure problems 2 and 4 leads, for $\mathrm{z}_{+}=1$, to:

$$
\begin{aligned}
& \frac{\mathrm{D}_{\mathrm{xx}}^{\mathrm{m}}}{\mathrm{D}_{\mathrm{s}}}=\frac{\mathrm{U}_{\mathrm{xx}}^{\mathrm{s}}}{\mathrm{D}_{\mathrm{s}}}=\frac{\left[4-\varepsilon_{\alpha}+\varepsilon_{\alpha} \Lambda_{\mathrm{w}}\right]-\mathrm{z}_{+} \varepsilon_{\alpha} \Lambda_{\mathrm{w}}+\mathrm{z}_{+} \varepsilon_{\alpha}}{2\left[\left(2-\varepsilon_{\alpha}\right)+\varepsilon_{\alpha} \Lambda_{\mathrm{w}}\right]}=\frac{2}{\left[\left(2-\varepsilon_{\alpha}\right)+\varepsilon_{\alpha} \Lambda_{\mathrm{w}}\right]} \\
& \frac{\mathrm{D}_{\mathrm{xx}}^{\mathrm{s}}}{\mathrm{D}_{\mathrm{s}}}=\frac{\mathrm{U}_{\mathrm{xx}}^{\mathrm{m}}}{\mathrm{D}_{\mathrm{s}}}=\frac{\left[4-\varepsilon_{\alpha}+\varepsilon_{\alpha} \Lambda_{\mathrm{w}}\right] \Lambda_{\mathrm{w}}-\mathrm{z}_{+} \varepsilon_{\alpha} \Lambda_{\mathrm{q}}^{2}}{2\left[\left(2-\varepsilon_{\alpha}\right)+\varepsilon_{\alpha} \Lambda_{\mathrm{w}}\right] \Lambda_{\mathrm{w}}} .
\end{aligned}
$$

In this case, we collect the surface contribution from the bulk coefficient with the 'migration' term, Eq. (87), according to:

$$
\begin{aligned}
& \frac{\mathrm{U}_{\mathrm{xx}}^{\mathrm{m} *}}{\mathrm{D}}=\frac{\varepsilon_{\alpha} \mathrm{U}_{\mathrm{eff}}^{\mathrm{bs}}}{\mathrm{D}}+\frac{\mathrm{U}_{\mathrm{xx}}^{\mathrm{m}}}{\mathrm{D}_{\mathrm{s}}} 2 \Lambda_{\mathrm{w}}\left(1-\varepsilon_{\alpha}\right), \\
& \frac{\mathrm{D}_{\mathrm{xx}}^{\mathrm{s} *}}{\mathrm{D}}=\frac{\mathrm{U}_{\mathrm{xx}}^{\mathrm{m} *}}{\mathrm{D}}=\frac{\left\{2 \varepsilon_{\alpha} \Lambda_{\mathrm{w}}+\left\{\left[4-\varepsilon_{\alpha}+\varepsilon_{\alpha} \Lambda_{\mathrm{w}}\right] \Lambda_{\mathrm{w}}-\mathrm{z}_{+} \varepsilon_{\alpha} \Lambda_{\mathrm{q}}^{2}\right\}\left(2-\varepsilon_{\alpha}\right)\right\}\left(1-\varepsilon_{\alpha}\right)}{\left[\left(2-\varepsilon_{\alpha}\right)+\varepsilon_{\alpha} \Lambda_{\mathrm{w}}\right]\left(2-\varepsilon_{\alpha}\right)} .
\end{aligned}
$$

These rearrangements lead to the following electrical potential closed equation:

$$
0=\underline{\nabla} \bullet \underline{\underline{U}}_{\text {eff }}^{*} \bullet\langle\mathrm{c}\rangle^{\alpha} \underline{\nabla}\langle\psi\rangle^{\alpha}+\underline{\nabla} \bullet \underline{\underline{U}}_{\text {eff }}^{\mathrm{s}^{*}} \bullet \underline{\nabla}\langle\mathrm{c}\rangle^{\alpha}-\mathrm{a}_{\mathrm{v}} \frac{\partial \mathrm{q}\rangle_{\alpha \beta}}{\partial \mathrm{t}} .
$$

Equations (85), (86), and (89) show that, due to the symmetry of the closure problems, we need to calculate only three out of the six transport coefficients defined in Eqns. (43) and (63).

\subsection{Spherical Unit Cell}

\subsubsection{Species Continuity Equation}

In this case, following Ochoa-Tapia et al. (1993), we can rewrite closure problem 1 as:

$$
\begin{aligned}
& \frac{2}{\mathrm{r}^{*}} \frac{\partial \mathrm{F}_{\mathrm{x}}}{\partial \mathrm{r}^{*}}+\frac{\partial^{2} \mathrm{~F}_{\mathrm{x}}}{\partial \mathrm{r}^{* 2}}+\frac{\cos \theta}{\mathrm{r}^{* 2} \sin \theta} \frac{\partial \mathrm{F}_{\mathrm{x}}}{\partial \theta}+\frac{1}{\mathrm{r}^{* 2}} \frac{\partial^{2} \mathrm{~F}_{\mathrm{x}}}{\partial \theta^{2}}+\frac{1}{\mathrm{r}^{* 2} \sin ^{2} \theta} \frac{\partial^{2} \mathrm{~F}_{\mathrm{x}}}{\partial \varphi^{2}}=0 \\
& -\frac{\partial \mathrm{F}_{\mathrm{x}}}{\partial \mathrm{r}^{*}}-\left(\frac{\mathrm{r}_{2}}{\mathrm{r}_{1}}\right) \Lambda_{\mathrm{w}}\left[\frac{\partial^{2} \mathrm{~F}_{\mathrm{x}}}{\partial \theta^{2}}+\frac{\cos \theta}{\sin \theta} \frac{\partial \mathrm{F}_{\mathrm{x}}}{\partial \theta}+\frac{1}{\sin ^{2} \theta} \frac{\partial^{2} \mathrm{~F}_{\mathrm{x}}}{\partial \varphi^{2}}\right]=\left(1-2 \Lambda_{\mathrm{w}}\right) \sin \theta \cos \varphi,(92) \\
& \text { at } \mathrm{r}^{*}=\mathrm{r}_{1} / \mathrm{r}_{2} \\
& \mathrm{~F}_{\mathrm{x}}=0, \text { at } \mathrm{r}^{*}=1
\end{aligned}
$$

The solution procedure detailed in the supplementary information (Appendix C) leads to:

$$
\frac{\varepsilon_{\alpha} \mathrm{D}_{\text {eff }}}{\mathrm{D}}=\frac{2 \varepsilon_{\alpha}+2 \Lambda_{\mathrm{w}} \varepsilon_{\alpha}}{\left(3-\varepsilon_{\alpha}\right)+2 \Lambda_{\mathrm{w}} \varepsilon_{\alpha}}
$$

Using Eq. (75), we can algebraically calculate the value of the surface contribution as:

$$
\frac{\varepsilon_{\alpha} \mathrm{D}_{\mathrm{eff}}^{\mathrm{bs}}}{\mathrm{D}}=\frac{6 \Lambda_{\mathrm{w}} \varepsilon_{\alpha}\left(1-\varepsilon_{\alpha}\right)}{\left(3-\varepsilon_{\alpha}\right)\left[\left(3-\varepsilon_{\alpha}\right)+2 \Lambda_{\mathrm{w}} \varepsilon_{\alpha}\right]} \text {. }
$$

A procedure similar to the one shown above leads to: 


$$
\begin{aligned}
& \frac{\mathrm{D}_{\mathrm{xx}}^{\mathrm{s}}}{\mathrm{D}_{\mathrm{s}}}=\frac{\left[9-\varepsilon_{\alpha}+2 \varepsilon_{\alpha} \Lambda_{\mathrm{w}}\right] \Lambda_{\mathrm{w}}-\mathrm{z}_{+} 4 \varepsilon_{\beta} \Lambda_{\mathrm{q}}^{2}}{3\left[\left(3-\varepsilon_{\alpha}\right)+2 \Lambda_{\mathrm{w}} \varepsilon_{\alpha}\right] \Lambda_{\mathrm{w}}}, \\
& \frac{\mathrm{D}_{\mathrm{xx}}^{\mathrm{m}}}{\mathrm{D}_{\mathrm{s}}}=\frac{9+3 \varepsilon_{\alpha}-2 \varepsilon_{\alpha} \Lambda_{\mathrm{w}}}{3\left[\left(3-\varepsilon_{\alpha}\right)+2 \Lambda_{\mathrm{w}} \varepsilon_{\alpha}\right]} .
\end{aligned}
$$

Eqns. (96) and (97) allow calculation of the value of the effective surface diffusivity and the value of the effective surface migration transport coefficient, respectively. Therefore, we can collect all the contributions to surface diffusion in a single surface diffusion transport coefficient $\left(D_{x x}^{1 s *}\right)$ given by:

$$
\begin{aligned}
\frac{\mathrm{D}_{\mathrm{xx}}^{\mathrm{b*} *}}{\mathrm{D}} & =\frac{\varepsilon_{\alpha} \mathrm{D}_{\text {eff }}^{\mathrm{bs}}}{\mathrm{D}}+\frac{\mathrm{D}_{\mathrm{xx}}^{\mathrm{s}}}{\mathrm{D}_{\mathrm{s}}} 3 \Lambda_{\mathrm{w}}\left(1-\varepsilon_{\alpha}\right), \\
\frac{\mathrm{D}_{\mathrm{xx}}^{\mathrm{s} *}}{\mathrm{D}} & =\frac{\left\{\left[\left(9-\varepsilon_{\alpha}+2 \varepsilon_{\alpha} \Lambda_{\mathrm{w}}\right) \Lambda_{\mathrm{w}}-\mathrm{z}_{+} 4 \varepsilon_{\beta} \Lambda_{\mathrm{q}}^{2}\right]\left(3-\varepsilon_{\alpha}\right)+6 \varepsilon_{\alpha} \Lambda_{\mathrm{w}}\right\}\left(1-\varepsilon_{\alpha}\right)}{3\left[\left(3-\varepsilon_{\alpha}\right)+2 \Lambda_{\mathrm{w}} \varepsilon_{\alpha}\right]\left(3-\varepsilon_{\alpha}\right)} .
\end{aligned}
$$

The arrangement of terms shown in Eq. (98) leads to the same closed species continuity equation appearing in Eq. (81). We can also calculate a global effective diffusivity using Eq. (46) to obtain:

$$
\begin{aligned}
& \frac{\varepsilon_{\alpha} \mathrm{D}_{\mathrm{xx}}^{*}}{\mathrm{D}}=\frac{\varepsilon_{\alpha} \mathrm{D}_{\mathrm{xx}}^{\mathrm{b}}}{\mathrm{D}}+\frac{\mathrm{D}_{\mathrm{xx}}^{\mathrm{s} *}}{\mathrm{D}} \\
& \frac{\varepsilon_{\alpha} \mathrm{D}_{\mathrm{xx}}^{*}}{\mathrm{D}}=\frac{\left[2 \varepsilon_{\alpha}+2 \Lambda_{\mathrm{w}} \varepsilon_{\alpha}\right]+\left\{\left[9-\varepsilon_{\alpha}+2 \varepsilon_{\alpha} \Lambda_{\mathrm{w}}\right] \Lambda_{\mathrm{w}}-\mathrm{z}_{+} 4 \varepsilon_{\beta} \Lambda_{\mathrm{q}}^{2}\right\}\left(1-\varepsilon_{\alpha}\right)}{\left[\left(3-\varepsilon_{\alpha}\right)+2 \Lambda_{\mathrm{w}} \varepsilon_{\alpha}\right]}
\end{aligned}
$$

Inspection of Eq. (101) shows that, if $\Lambda_{\mathrm{w}} \ll<1$ and $\Lambda_{\mathrm{q}}<<1$, we obtain Maxwell's (1881) solution for the bulk effective diffusivity.

$$
\frac{\varepsilon_{\alpha} \mathrm{D}_{\mathrm{xx}}^{\mathrm{b}}}{\mathrm{D}}=\frac{2 \varepsilon_{\alpha}}{\left(3-\varepsilon_{\alpha}\right)} .
$$

Similar solution was obtained by Ochoa-Tapia et al. (1993) for the case of uncharged porous media.

\subsubsection{Electrical Potential}

The symmetry conditions described in the previous sections also apply here; therefore, we have:

$$
\begin{aligned}
& \frac{\varepsilon_{\alpha} \mathrm{D}_{\mathrm{xx}}^{\mathrm{bs}}}{\mathrm{D}}=\frac{\varepsilon_{\alpha} \mathrm{U}_{\mathrm{xx}}^{\mathrm{bs}}}{\mathrm{D}}=\frac{6 \varepsilon_{\alpha} \Lambda_{\mathrm{w}}\left(1-\varepsilon_{\alpha}\right)}{\left[\left(3-\varepsilon_{\alpha}\right)+2 \varepsilon_{\alpha} \Lambda_{\mathrm{w}}\right]\left(3-\varepsilon_{\beta}\right)} \\
& \frac{\mathrm{D}_{\mathrm{xx}}^{\mathrm{ls} *}}{\mathrm{D}}=\frac{\mathrm{U}_{\mathrm{xx}}^{\mathrm{m} *}}{\mathrm{D}}=\frac{\left\{\left[\left(9-\varepsilon_{\alpha}+2 \varepsilon_{\alpha} \Lambda_{\mathrm{w}}\right) \Lambda_{\mathrm{w}}-\mathrm{z}_{+} 4 \varepsilon_{\beta} \Lambda_{\mathrm{q}}^{2}\right]\left(3-\varepsilon_{\alpha}\right)+6 \varepsilon_{\alpha} \Lambda_{\mathrm{w}}\right\}\left(1-\varepsilon_{\alpha}\right)}{\left[\left(3-\varepsilon_{\alpha}\right)+2 \Lambda_{\mathrm{w}} \varepsilon_{\alpha}\right]\left(3-\varepsilon_{\alpha}\right)} \\
& \frac{\mathrm{D}_{\mathrm{xx}}^{\mathrm{m} *}}{\mathrm{D}}=\frac{\mathrm{U}_{\mathrm{xx}}^{\mathrm{s} *}}{\mathrm{D}}=\frac{9+3 \varepsilon_{\alpha}-2 \varepsilon_{\alpha} \Lambda_{\mathrm{w}}}{\left[\left(3-\varepsilon_{\alpha}\right)+2 \Lambda_{\mathrm{w}} \varepsilon_{\alpha}\right]} \Lambda_{\mathrm{q}}\left(1-\varepsilon_{\alpha}\right)
\end{aligned}
$$

These rearrangements lead to the same electrical potential closed balance given by Eq. (90).

\section{RESUltS}

\subsection{Evaluation of the Model's Constraints}


The constraints defined in Eq. (27) play a critical role in defining the applicability of the theory presented in this work. Therefore, we estimated their value in common applications. First, we transformed the constraints given in Eq. (27) into dimensionless forms.

$$
\left(2 \zeta \frac{\left\langle\mathrm{w}^{*}\right\rangle_{\alpha \beta}}{\left\langle\mathrm{c}^{*}\right\rangle^{\alpha}}\right)<<1,\left(2 \zeta \frac{\left\langle\mathrm{q}^{*}\right\rangle_{\alpha \beta}\langle\psi\rangle^{\alpha}}{\left\langle\mathrm{c}^{*}\right\rangle^{\alpha}}\right)<<1,\left(2 \zeta \frac{\mathrm{D}_{\mathrm{s}}\left\langle\mathrm{w}^{*}\right\rangle_{\alpha \beta}}{\mathrm{D}\left\langle\mathrm{c}^{*}\right\rangle^{\alpha}}\right)<<1,\left(2 \zeta \frac{\mathrm{D}_{\mathrm{s}}\left\langle\mathrm{q}^{*}\right\rangle_{\alpha \beta}\langle\psi\rangle^{\alpha}}{\mathrm{D}\left\langle\mathrm{c}^{*}\right\rangle^{\alpha}}\right)<<1 .(106)
$$

Here, $\zeta$ is the small parameter defined as $\zeta=\lambda_{D} / L_{c}$.

Estimates were calculated using typical experimental values for the CDI process: electrode thickness $\left(\mathrm{L}_{\mathrm{e}}\right)=0.001 \mathrm{~m}, \mathrm{D}=1.510^{-9} \mathrm{~m}^{2} / \mathrm{s}$, pore diameter $=50 \mathrm{~nm}$, void fraction $=0.5$, salt concentration $=0.05 \mathrm{M}$, half-electrode potential $(\phi)=0.5 \mathrm{~V}$ corresponding to the dimensionless values, $\psi=20, \psi_{\mathrm{D}} \cong 16$, and the characteristic time $(\tau)$ equal to 11.1 minutes. According to the GCS model, for large pores, we used the following expressions to evaluate the dimensionless ratios:

$$
\begin{aligned}
& \frac{\left\langle\mathrm{w}^{*}\right\rangle_{\alpha \beta}}{\left\langle\mathrm{c}^{*}\right\rangle^{\alpha}}=\frac{4}{\sqrt{\left\langle\mathrm{c}^{*}\right\rangle^{\alpha}}} \sinh ^{2}\left(\Delta \psi_{\mathrm{D}} / 4\right), \\
& \frac{\left\langle\mathrm{q}^{*}\right\rangle_{\alpha \beta}\langle\psi\rangle^{\alpha}}{\left\langle\mathrm{c}^{*}\right\rangle^{\alpha}}=\frac{2\langle\psi\rangle^{\alpha}}{\sqrt{\left\langle\mathrm{c}^{*}\right\rangle^{\alpha}}} \sinh \left(\Delta \psi_{\mathrm{D}} / 2\right) .
\end{aligned}
$$

The estimate leads to $0.004<<1,0.08<<1,0.001<<1,0.02<<1$.

In conclusion, we can say that, in most practical situations, the constraints given by Eq. (27) are acceptable and so the equations derived in this work.

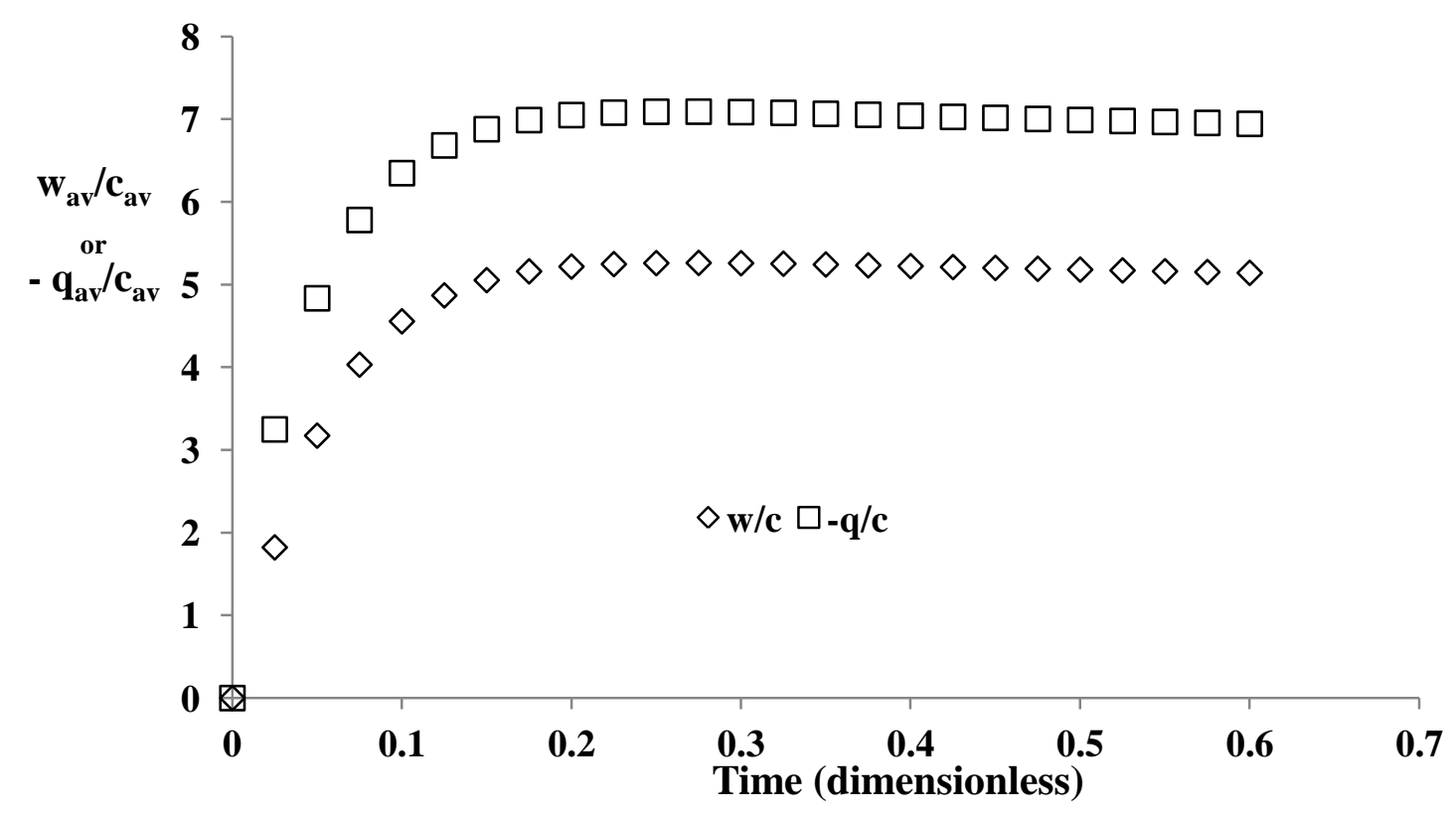

Figure 3. Variation of the ratios $\mathrm{w}_{\mathrm{av}} / \mathrm{c}_{\mathrm{av}}$ and $-\mathrm{q}_{\mathrm{av}} / \mathrm{c}_{\mathrm{av}}$ with time. 
In Figure 3, we plot the value of the ratios $\mathrm{w}_{\mathrm{av}} / \mathrm{c}_{\mathrm{av}}$ and $-\mathrm{q}_{\mathrm{av}} / \mathrm{c}_{\mathrm{av}}$ versus time. These ratios represent the average value of $\langle\mathrm{w}\rangle_{\alpha \beta} /\langle\mathrm{c}\rangle^{\alpha}$ and $-\langle\mathrm{q}\rangle_{\alpha \beta} /\langle\mathrm{c}\rangle^{\alpha}$ calculated along the whole electrode length $\left(\mathrm{L}_{\mathrm{e}}\right)$. These results have been calculated using a modification of the computer code developed by Sharma et al. (2013). This computer code was modified to solve equations (89) and (95). More details about the code can be found in the reference.

Figure 3 shows that both average ratios increase continuously during the supercapacitor regime (short times), and decrease slightly until becoming constant at long times (desalination regime). The value of the electrode charge efficiency can be determined by calculation of the $\mathrm{w}_{\mathrm{av}} /-\mathrm{q}_{\mathrm{av}}$ ratio. The values of the $\Lambda_{\mathrm{w}}$ and $\Lambda_{\mathrm{q}}$ parameters are calculated using:

$$
\Lambda_{\mathrm{w}}=2 \frac{\lambda_{\mathrm{D}}}{\mathrm{r}_{1}} \frac{\mathrm{D}_{\mathrm{s}}}{\mathrm{D}} \frac{\mathrm{w}_{\mathrm{av}}}{\mathrm{c}_{\mathrm{av}}}, \Lambda_{\mathrm{q}}=2 \frac{\lambda_{\mathrm{D}}}{\mathrm{r}_{1}} \frac{\mathrm{D}_{\mathrm{s}}}{\mathrm{D}} \frac{\mathrm{q}_{\mathrm{av}}}{\mathrm{c}_{\mathrm{av}}} .
$$

Therefore, the time variation of $\mathrm{w}_{\mathrm{av}} / \mathrm{c}_{\mathrm{av}}$ and $-\mathrm{q}_{\mathrm{av}} / \mathrm{c}_{\mathrm{av}}$ ratios produces similar variation of the $\Lambda_{\mathrm{w}}$ and $\Lambda_{\mathrm{q}}$ parameters with time. The $\Lambda_{\mathrm{w}}$ and $\Lambda_{\mathrm{q}}$ parameters also vary with position inside the electrode.

An important issue is the evaluation of the ratio $d=D_{s} / D$ appearing in Eq. (110). Ochoa-Tapia et al. (1993) assumed that the ratio $d \leq 1$; however, no values were given. In Table I, we show some indicative values taken from Revil (1999).

Table I. Ionic surface diffusivities in clay (Data from ref. 41).

\begin{tabular}{|c|c|c|c|}
\hline Ion & $D\left(\mathrm{~m}^{2} \mathrm{~s}^{-1}\right)$ & $D_{S}\left(\mathrm{~m}^{2} \mathrm{~s}^{-1}\right)$ & $D_{S} / D$ \\
\hline \hline $\mathrm{H}^{+}$ & $9.31 \mathrm{E}-9$ & $4.11 \mathrm{E}-10$ & 0.044 \\
\hline $\mathrm{Li}^{+}$ & $1.03 \mathrm{E}-9$ & $1.29 \mathrm{E}-10$ & 0.125 \\
\hline $\mathrm{Na}^{+}$ & $1.33 \mathrm{E}-9$ & $1.31 \mathrm{E}-10$ & 0.098 \\
\hline $\mathrm{K}^{+}$ & $1.96 \mathrm{E}-9$ & $0.95 \mathrm{E}-10$ & 0.048 \\
\hline $\mathrm{NH}^{+}$ & $1.96 \mathrm{E}-9$ & $0.59 \mathrm{E}-10$ & 0.030 \\
\hline $\mathrm{Rb}^{+}$ & $2.07 \mathrm{E}-9$ & $0.51 \mathrm{E}-10$ & 0.027 \\
\hline $\mathrm{Cs}^{+}$ & $2.06 \mathrm{E}-9$ & $0.39 \mathrm{E}-10$ & 0.019 \\
\hline
\end{tabular}

In Table I, D is the ionic diffusivity in water at infinite dilution, while $\mathrm{D}_{\mathrm{s}}$ is the surface diffusivity in clay determined from experimental data using the model presented by Revil (1999). In all our calculations we used a value of $d=0.2$.

The variation of the effective surface diffusivity, effective surface mobility, and global effective diffusivity values with void fraction $\left(\varepsilon_{\alpha}\right)$ are shown in Figures 4, 5, and 6, respectively. The results presented in these Figures were calculated using typical experimental values for the CDI process: electrode thickness $\left(\mathrm{L}_{\mathrm{e}}\right)=0.001 \mathrm{~m}, \mathrm{D}=1.510^{-9} \mathrm{~m}^{2} / \mathrm{s}$, pore diameter $=40 \mathrm{~nm}$, salt concentration $=0.1 \mathrm{M}$, half-electrode potential $=0.25 \mathrm{~V}$ corresponding to $\psi=10$, and $\mathrm{d}=0.2$.

It is shown in Figure 4 that the effective surface diffusivity increases continuously as the void fraction increases, reaches a maximum at $\varepsilon_{\alpha} \cong 0.9$, and for higher $\varepsilon_{\alpha}$ decreases until approaching 0 at $\varepsilon_{\alpha}=1$. Similar behavior is observed in Figure 5. The effective surface mobility increases with the porosity until it reaches a maximum value $\left(\varepsilon_{\alpha} \cong 0.9\right)$ and, for bigger porosity values, the effective surface mobility decreases until approaching 0 at $\varepsilon_{\alpha}=1$. The effective mobility depends 
upon the excess charge $(\mathrm{q})$; therefore, it could take negative values when the electrode charge is positive.

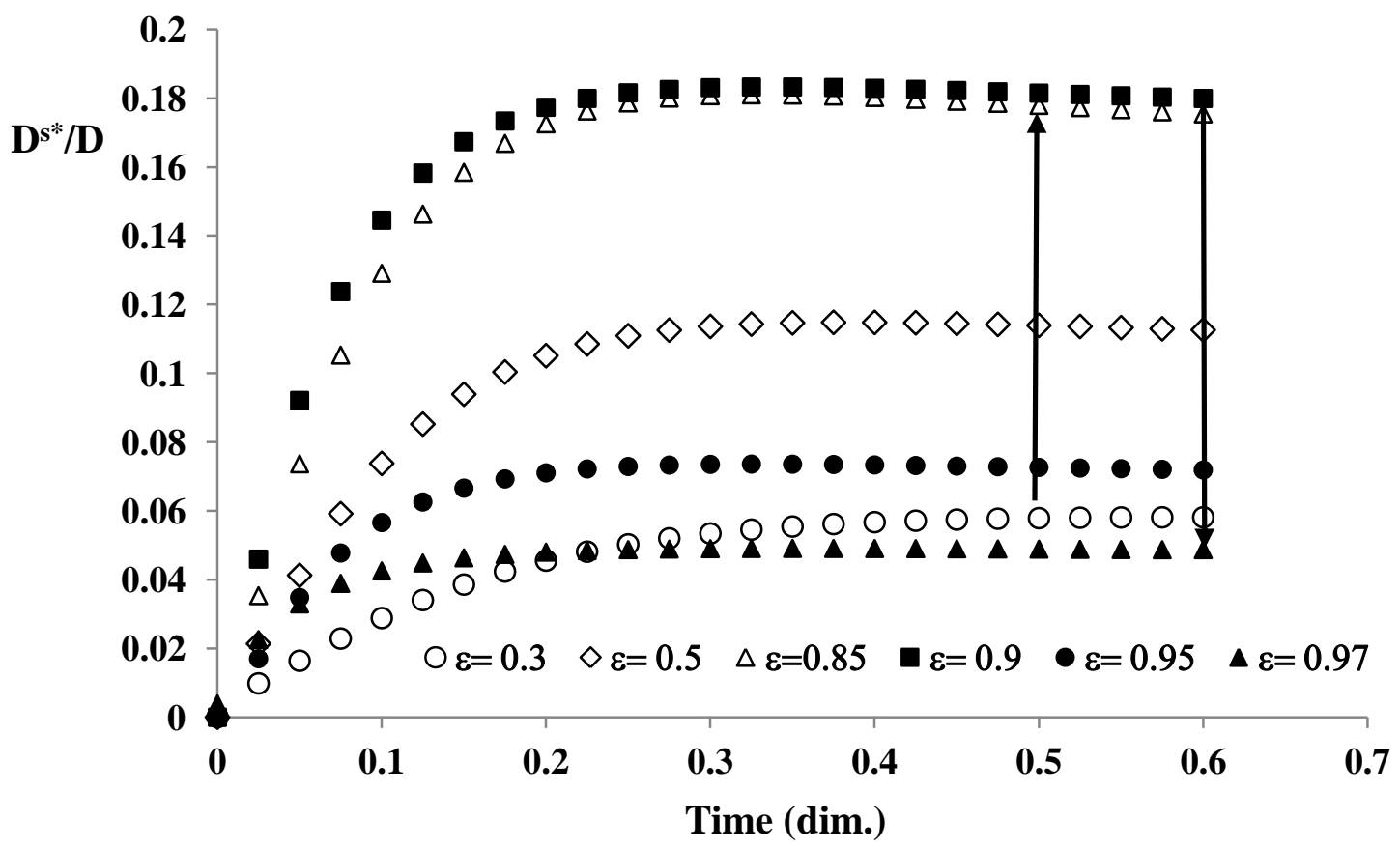

Figure 4. Time variation of effective surface diffusivity in beds of infinite cylindrical particles.

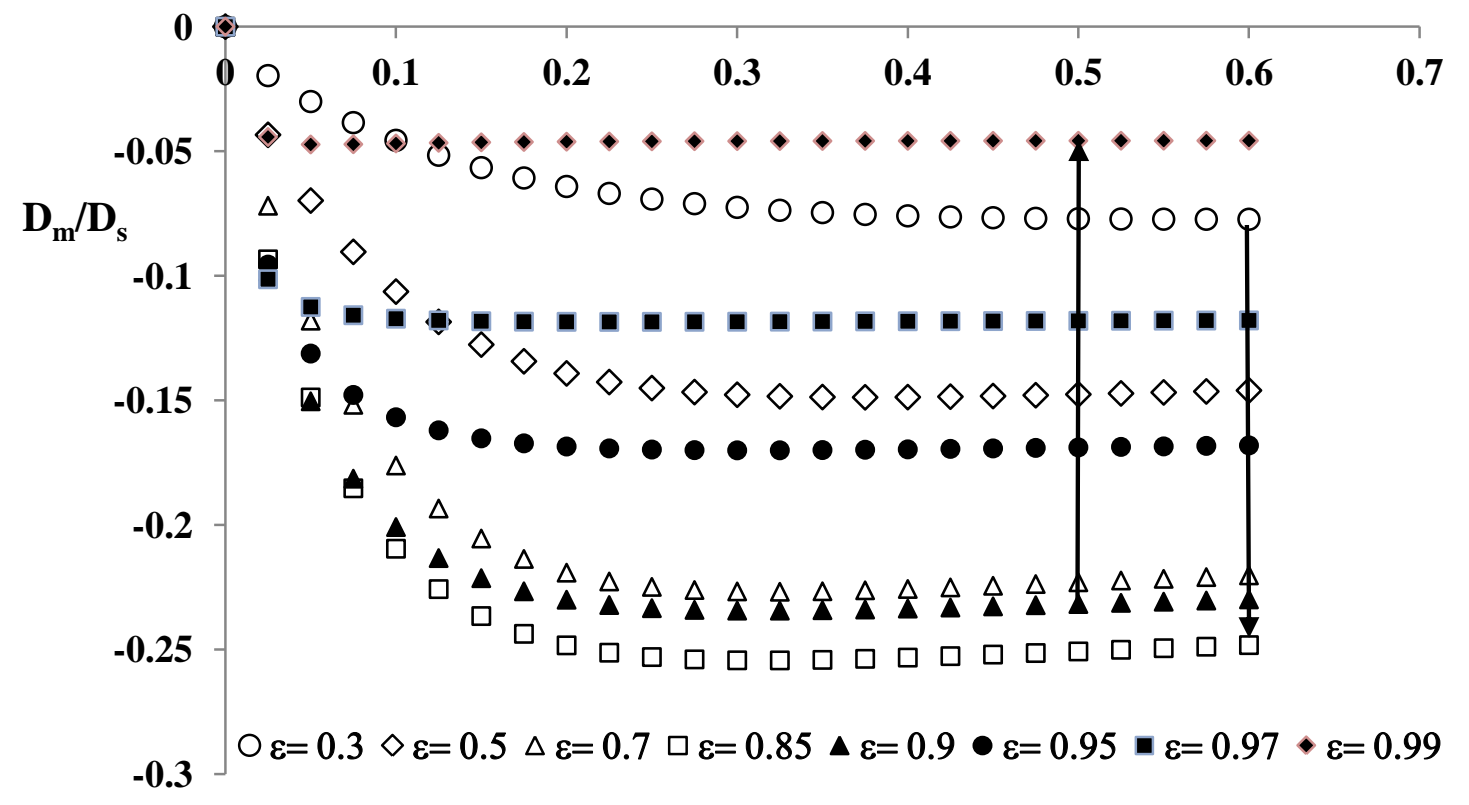

\section{Time (dim.)}

Figure 5. Time variation of effective surface mobility in beds of infinite cylindrical particles.

The increase of the surface diffusivities with void fraction is similar to the behavior reported by many authors (Ho and Strider, 1981; Ochoa-Tapia et al., 1993; among others). However, at the $\varepsilon_{\alpha}=1$ limit, global diffusivity $\left(\mathrm{D}_{\mathrm{xx}}^{*}\right.$ ) values tend to the bulk diffusivity values and the surface 
diffusivity $\left(\mathrm{D}_{\mathrm{xx}}^{\mathrm{s}^{*}}\right.$ ) tends to 0 . The decrease in value of the surface transport coefficients at high void fraction values produces the presence of the maxima in the surface transport coefficients curves shown in Figures 4 and 5. Similar behavior can be observed in the results reported by Ho and Strider (1981) and Ochoa-Tapia et al. (1993) for uncharged porous media.

In Figure 6 we plot the value of the combined effective diffusivity defined in Eq. (86) as a function of void fraction. We can see in Figure 6 that the combined effective diffusivity continuously increases as the void fraction increases until, at $\varepsilon_{\alpha}=1$, is equal to the bulk solution diffusivity.

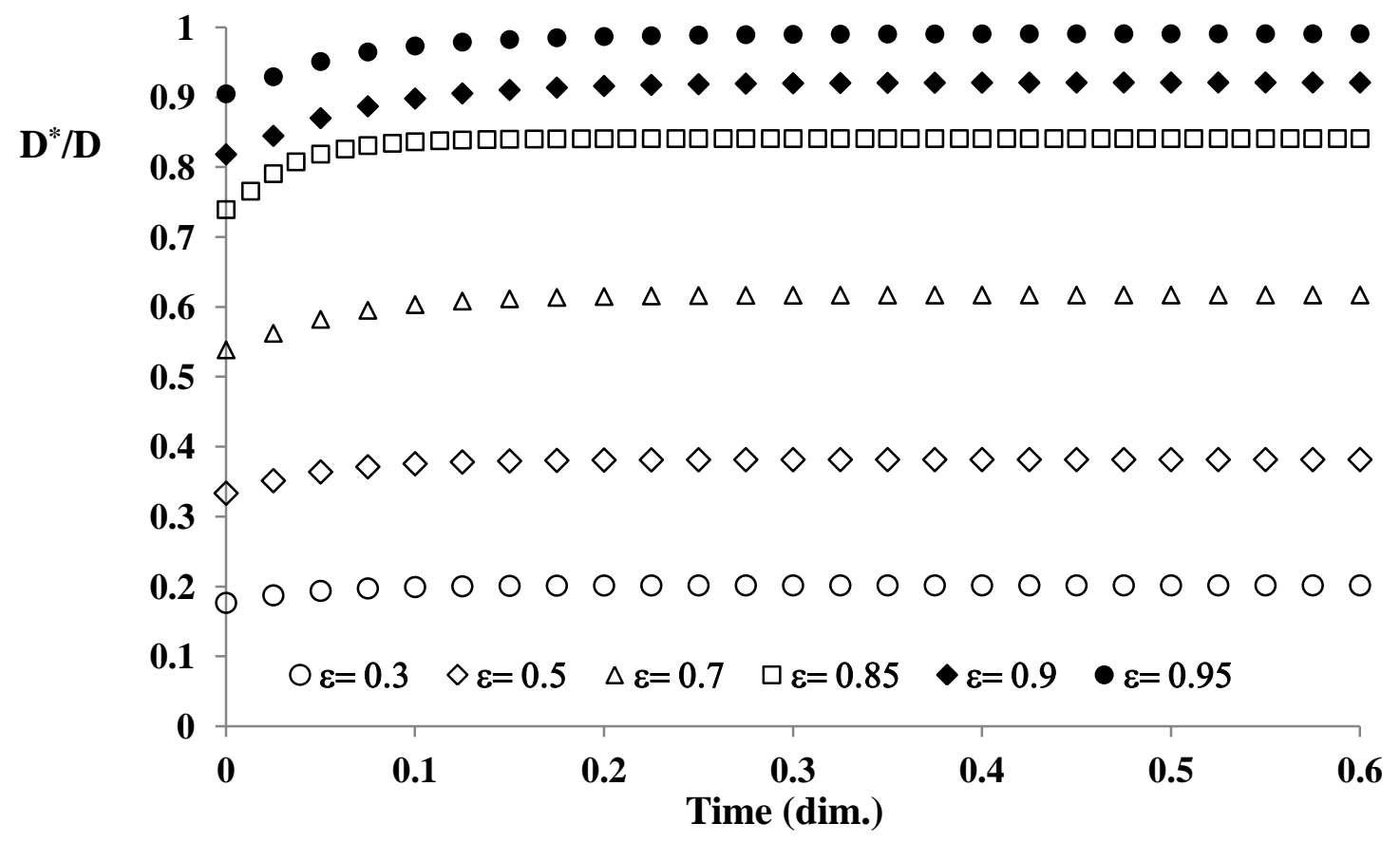

Figure 6. Time variation of the combined effective diffusivity.

Following the procedure described in the closure problem solution, we also calculated the value for the effective transport parameters in beds of spherical particles. In all our calculations, not shown here, the values for beds of spherical particles are smaller than the corresponding values for cylinders. This effect is produced because, for spheres at the same void fraction, the particle radius $\left(r_{1}\right)$ is bigger than the corresponding cylinder value. Therefore, the $\Lambda_{i}$ parameters for spheres are smaller than the corresponding ones for cylinders.

In Figure 7 we compare our results with the theoretical results reported by Ochoa-Tapia et al. (1993) for uncharged porous media. Intuitively, we associate the parameter $\Lambda_{\mathrm{w}}$ with surface diffusion due to concentration gradients, while the parameter $\Lambda_{\mathrm{q}}$ represents the influence of charge gradients. Therefore, in Figure 7 we plot Eq. (83) and Eq. 5.15 in Ochoa-Tapia et al. (1993). In order to compare values for charged and uncharged porous media we set $\Lambda_{\mathrm{q}}$ to zero in Eq. (83) and replaced $\Lambda_{\mathrm{w}}$ by the parameter $\Lambda$ used by Ochoa-Tapia et al. (1993). Incidentally, in Ochoa-Tapia et al. (1993) original article there is a typing error in Eq. 5.15. This equation should be given by: 


$$
\frac{\varepsilon_{\alpha} \mathrm{D}_{\mathrm{xx}}^{*}}{\mathrm{D}}=\frac{\varepsilon_{\alpha}\left[\left(2-\varepsilon_{\alpha}\right)+\varepsilon_{\alpha} \Lambda_{\mathrm{w}}-\left(1-\Lambda_{\mathrm{w}}\right)^{2}\left(1-\varepsilon_{\alpha}\right)\right]}{\left[\left(2-\varepsilon_{\beta}\right)+\varepsilon_{\beta} \Lambda_{\mathrm{w}}\right]}+2\left(1-\varepsilon_{\alpha}\right) \Lambda_{\mathrm{w}} .
$$

Figure 7 shows that equation (83) derived in this work and Eq. 5.15 presented in OchoaTapia et al. (1993) produce identical results. It can also be concluded that the surface diffusivity term appearing in Eq. (89) is equivalent to similar terms appearing in mass transport in uncharged porous media, while the surface mobility process is unique to charged porous media. Similar conclusions were drawn when plotting the values calculated for spherical particles. Numerical results, not included here, also show that Eq. (101) is identical to Eq. 5.18 in OchoaTapia et al. (1993).

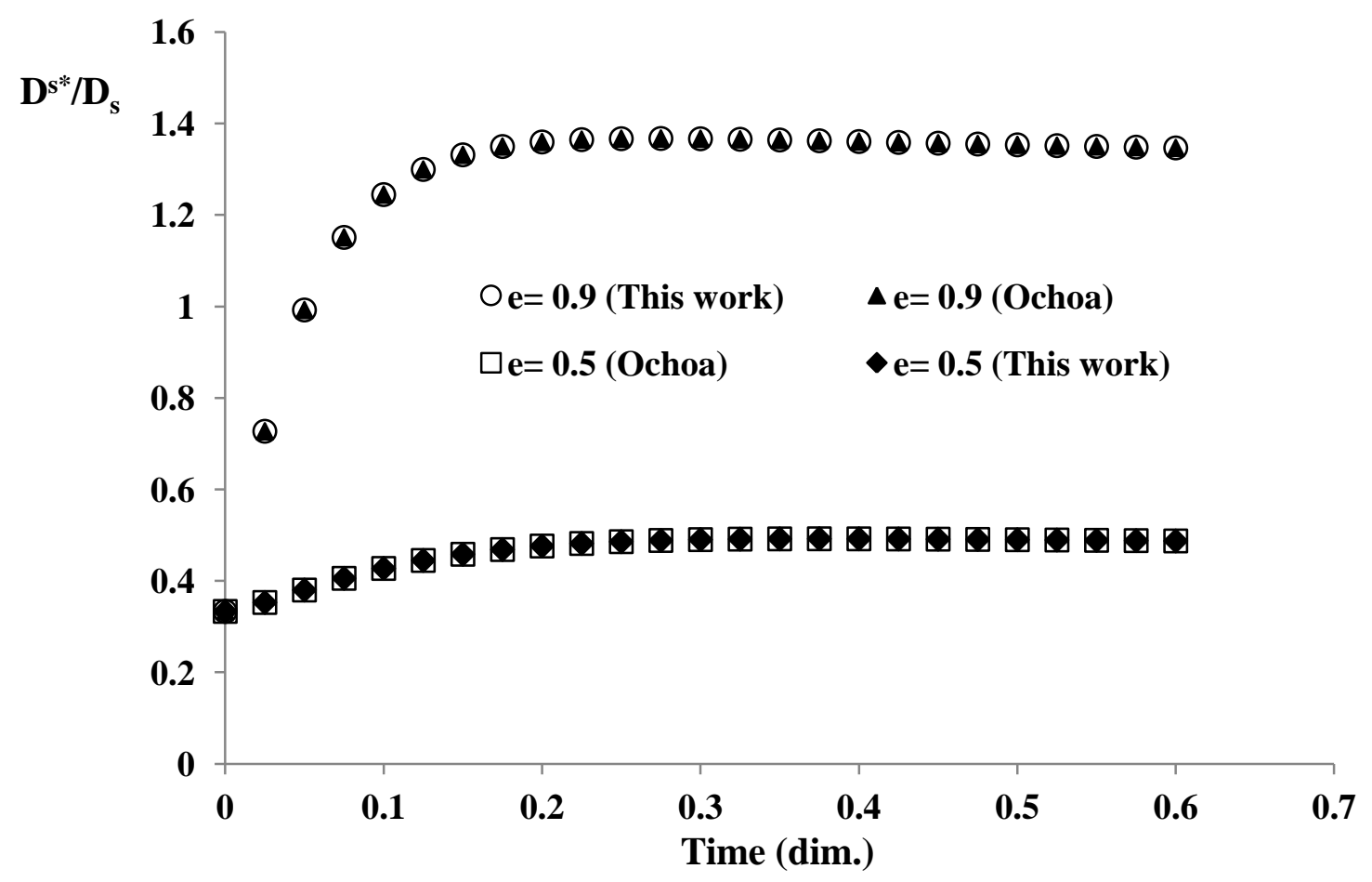

Figure 7. Comparison of results for charged and uncharged porous media.

Volfkovich et al. (2013) reported the following equation relating conductivities:

$$
\kappa(c, \phi)=\kappa(c)+\kappa_{s c h}(\phi)+\kappa_{\mathrm{sg}}(\mathrm{q}) \text {. }
$$

Equations (43) and (67) can be re-arranged as,

$$
\begin{aligned}
& \varepsilon_{\alpha} \frac{\partial \mathrm{c}\rangle^{\alpha}}{\partial \mathrm{t}}=\underline{\nabla} \bullet\left\{\left[\varepsilon_{\alpha} \underline{\underline{\kappa}}+\mathrm{a}_{\mathrm{v}} \underline{\underline{\kappa}}_{\mathrm{M}}\right] \bullet \underline{\nabla} \ln \langle\mathrm{c}\rangle^{\alpha}\right\}+\mathrm{z}_{+} \underline{\nabla} \bullet\left\{\mathrm{a}_{\mathrm{v}} \underline{\underline{\kappa}}_{\mathrm{D}} \bullet \underline{\nabla}\langle\psi\rangle^{\alpha}\right\}-\mathrm{a}_{\mathrm{v}} \frac{\partial\langle\mathrm{w}\rangle_{\alpha \beta}}{\partial \mathrm{t}}, \\
& \underline{\nabla} \bullet\left\{\left[\varepsilon_{\alpha} \underline{\underline{\kappa}}+\mathrm{z}_{+} \mathrm{a}_{\mathrm{v}} \underline{\underline{\kappa}}_{\mathrm{M}}\right] \bullet \underline{\nabla}\langle\psi\rangle^{\alpha}+\mathrm{a}_{\mathrm{v}} \underline{\underline{\kappa}}_{\mathrm{D}} \bullet \underline{\nabla} \ln \langle\mathrm{c}\rangle^{\alpha}\right\}-\mathrm{a}_{\mathrm{v}} \frac{\partial \mathrm{q}\rangle_{\alpha \beta}}{\partial \mathrm{t}}=0 . \text { (114) }
\end{aligned}
$$

In the derivation of equations (113) and (114), we have used the following definitions based upon the symmetric characteristics of the transport parameters:

$$
\underline{\underline{\kappa}}=\underline{\underline{D}}_{\text {eff }}\langle\mathrm{c}\rangle^{\alpha}=\underline{\underline{U}}_{\text {eff }}\langle\mathrm{c}\rangle^{\alpha}, \underline{\underline{\kappa}}_{\mathrm{M}}=\underline{\underline{\mathrm{D}}}_{\mathrm{eff}}^{\mathrm{s}}\langle\mathrm{w}\rangle_{\alpha \beta}=\underline{\underline{\mathrm{U}}}_{\mathrm{eff}}^{\mathrm{m}}\langle\mathrm{w}\rangle_{\alpha \beta}, \underline{\underline{\kappa}}_{\mathrm{D}}=\underline{\underline{D}}_{\mathrm{eff}}^{\mathrm{m}}\langle\mathrm{q}\rangle_{\alpha \beta}=\underline{\underline{\mathrm{U}}}_{\mathrm{eff}}^{\mathrm{s}}\langle\mathrm{q}\rangle_{\alpha \beta} .
$$


Here, $\stackrel{\kappa}{=}$ represents the bulk conductivity, $\underline{\underline{M}}_{\mathrm{M}}$ the conductivity due to surface potential gradients, and $\underline{\underline{\kappa}}_{\mathrm{D}}$ is the conductivity due to surface diffusion gradients. In equations (113) and (114), we can defined a global conductivity $\left(\underline{\underline{\kappa}}^{*}\right)$ given by,

$$
\underline{\underline{\kappa}}^{*}=\varepsilon_{\alpha} \underline{\underline{\kappa}}+\mathrm{z}_{+} \mathrm{a}_{\mathrm{v}} \underline{\underline{\kappa}} \mathrm{M} \text {. }
$$

The global conductivity is equivalent to the classical conductivity in free electrolytes when there are no significant concentration gradients (Newman and Thomas-Alyea, 2004). The global conductivity has contributions from both bulk $(\underline{\underline{\kappa}})$ and surface transport processes $(\underset{\underline{\kappa}}{\underline{\kappa}})$. In cases where there are significant concentration gradients a surface diffusion contribution to the conductivity appears $\left(\underline{\underline{\kappa}}_{\mathrm{D}}\right)$. A comparison of equations (113) and (114) derived in this work and Eq. (112) from Volfkovich et al. (2013) shows that, these authors are considering the right contributions to ionic transport. However, the surface transport parameter depends upon w and not q.

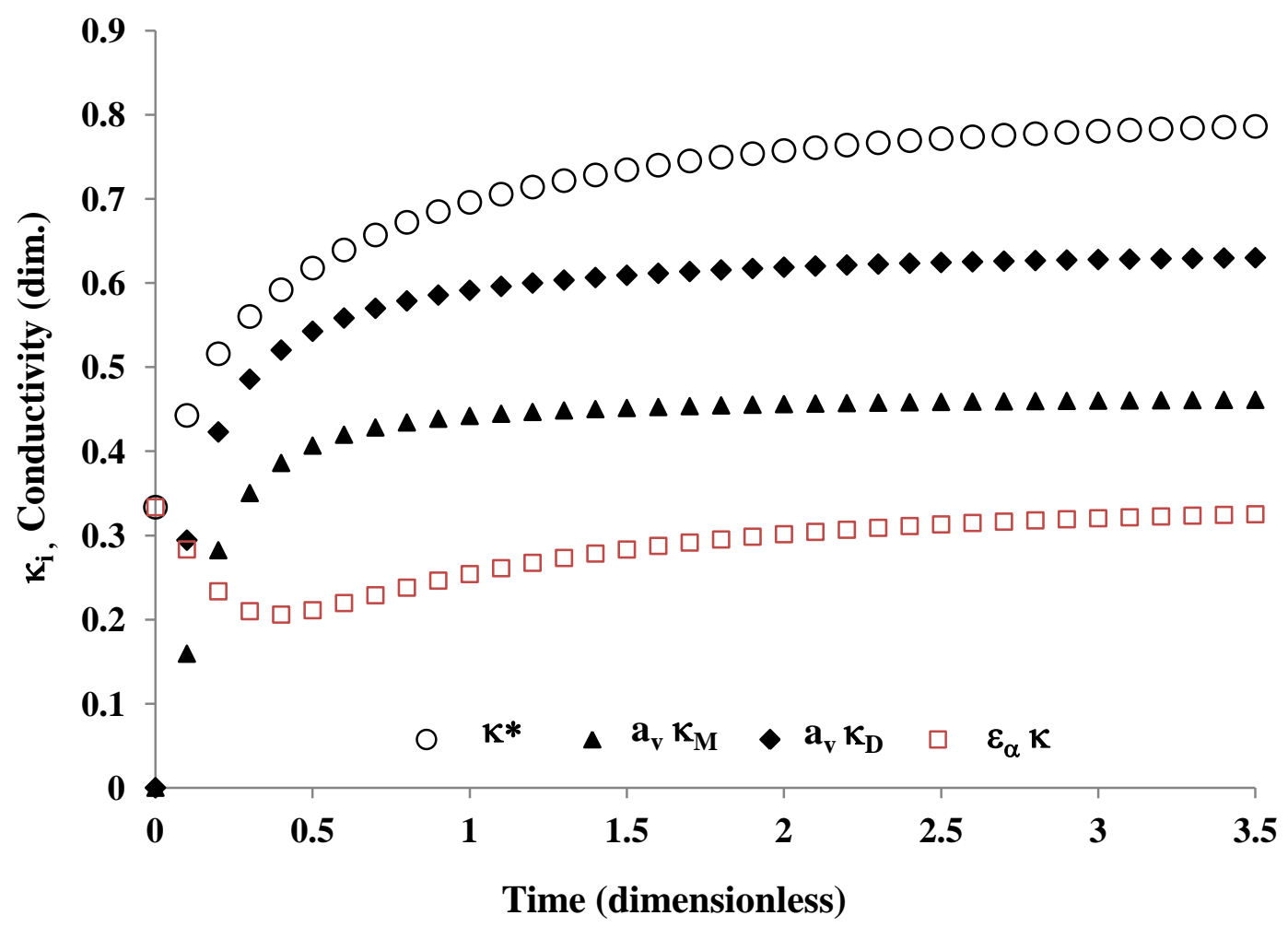

Figure 8. Comparison of different dimensionless contributions to total conductivity for $\mathrm{C}_{\mathrm{o}}=$ $0.005 \mathrm{M}$.

In Figure 8 we plot the dimensionless global $\left(\kappa^{*}\right)$, the bulk $\left(\varepsilon_{\alpha} \underline{\underline{\kappa}}\right)$, surface diffusion $\left(\mathrm{a}_{\mathrm{v}} \underline{\underline{\kappa}}_{\mathrm{D}}\right)$, and surface migration $\left(\mathrm{a}_{\mathrm{v}} \underline{\underline{\kappa}}_{\mathrm{M}}\right)$ conductivities versus time for a low salinity value $\left(\mathrm{C}_{\mathrm{o}}=0.005 \mathrm{M}\right)$. In our calculations we used $\mathrm{z}_{+}=1$. We can see in Figure 8 that the bulk conductivity $\left(\varepsilon_{\alpha} \underline{\underline{\kappa}}\right)$ during the supercapacitor regime (short times) decreases while at longer times (desalination regime) it slowly increases until reaching saturation values. This behavior is produced by the 
average salt concentration variation during the charging cycle. At short times, salt concentration inside the pores decreases in value because ionic transport into the EDLs is faster than ion replenishment by diffusion from outside the electrode. As the EDLs become saturated the ionic transfer into the EDL decreases and the diffusivity from free electrolyte increases. At long times the diffusive flow slowly increases the salt concentration inside the pores. This is not the case for the surface transport parameters which depend upon the values of $w$ or $q$ inside the EDLs. These values increase monotonically from 0 until reaching saturation values. It is also shown in the figure that at short times the bulk contribution to the global conductivity is higher than the surface ones; however, as time increases the surface contributions become higher than the bulk one. Our results support Volfkovich et al. (2013) conclusion that as the concentration tends to zero the surface migration conductivity $\left(\mathrm{a}_{\mathrm{v}} \underline{\underline{\kappa}}_{\mathrm{M}}\right)$ becomes the main contribution to global conductivity. The same numerical results demonstrate that the diffusion conductivity $\left(\mathrm{a}_{\mathrm{v}} \underline{\underline{\kappa}}_{\mathrm{D}}\right)$ also increases as the concentration decreases. In all our calculations the values of the surface diffusion conductivity were found to be higher than the equivalent surface migration conductivity ones. However, the surface diffusion term will contribute less to ionic transport because the term $\underline{\nabla}$ lnc is normally smaller than $\underline{\nabla} \phi$. The results shown in Figure 8 were calculated using the same operating values used in Figures 4, 5, and 6. Dimensional conductivity values can be calculated by multiplying the dimensionless values by $0.0579 \mathrm{~S} / \mathrm{m}\left(2 \mathrm{~F} \mathrm{D}_{\mathrm{o}} \mathrm{C}_{\mathrm{o}} /\right.$ $\mathrm{V}_{\mathrm{T}}$ ), for example, in Figure 8 the steady state dimensional values are: $\kappa^{*}=0.0456 \mathrm{~S} / \mathrm{m}, \varepsilon_{\alpha} \underline{\underline{\kappa}}=$ $0.0189 \mathrm{~S} / \mathrm{m}, \mathrm{a}_{\mathrm{v}} \underline{\underline{\kappa}}_{\mathrm{D}}=0.0365 \mathrm{~S} / \mathrm{m}$, and $\mathrm{a}_{\mathrm{v}} \underline{\underline{\kappa}}_{\mathrm{M}}=0.0267 \mathrm{~S} / \mathrm{m}$. All these values are of the same order of magnitude than the experimental value for free solution, $0.0601 \mathrm{~S} / \mathrm{m}$.

Revil (1999) also discussed the different mechanisms that produced surface transport. Using experimental data, he concluded that surface transport occurs mainly by movement inside the Stern layer, while ion movement inside the diffuse layer is negligible. These findings led him to report that surface conductivity occurs mainly through an electromigration mechanism and there is no significant concentration driven mechanism. However, in this work inspection of Eq. (114) shows that this occurs only when the value of the term $\underline{\nabla} \operatorname{lnc}$ is very small. This conclusion is supported by the results presented in Figure 8.

\section{CONCLUSIONS}

The volume averaging method has been used to derive the averaged equations describing bulk and surface transport phenomena in charged porous electrodes during electrosorption. We have derived the complete form of the volume averaged equations starting from the point equations and the appropriate boundary conditions. We have used the surface averaging theorem to deal with the surface transport terms. As a result of the homogenization technique, two extra surface transport terms appear in the averaged equations. One term describes surface diffusion and the other surface migration. Surface and bulk diffusion can be lumped together in a single term. A combined global effective diffusivity controls the combined diffusion process. Our theoretical derivations support the characterization of surface transport phenomena by the use of a surface migration term plus a combined effective diffusivity. 
We have calculated bulk, surface, and combined effective diffusivities for isotropic porous media of cylinders and spheres by solving the closure problem in Chang's unit cells. Our results for the combined effective diffusivity are similar to the ones obtained for uncharged porous media by Ochoa-Tapia et al. (1993). However, the influence of the charge precludes obtaining constant transport parameters during the supercapacitor regime. In all cases, the charge increases the value of the transport parameters. The simulation results presented in this work show that there are two non-negligible contributions to surface conductivity. There is a concentration gradient contribution similar to the transport process in uncharged porous media and a surface electromigration contribution produced by the presence of the charged porous medium.

\section{ACKNOWLEDGMENTS}

This research was partially conducted at the Oak Ridge National Laboratory (ORNL). ORNL is managed by UT-Battelle, LLC, under Contract DE-AC05-0096OR22725 with the U.S. Department of Energy.

\section{REFERENCES}

1. Bazant, M. Z., Thornton, K., and Ajdari, A. "Diffuse-Charge Dynamics in Electrochemical Systems," Phys. Rev. E, 70, 021506, 2004.

2. Bikerman, J. J. "Electrokinetic Equations and Surface Conductance. A Survey of the Diffuse Double Layer Theory of Colloidal Solutions," Trans. Faraday Soc., 36, 154-160, 1940.

3. Biesheuvel, P. M., and Bazant, M. Z. "Nonlinear Dynamics of Capacitive Charging and Desalination by Porous Electrodes," Phys. Rev. E, 81, 031502, 2010.

4. Bockris, J. O., Reddy, A. K. N., and Gamboa-Aldeco, M. "Modern Electrochemistry: Fundamentals of Electrodics," Vol. 2b, Plenum Pub. Corp, 2000.

5. Borges da Silva, E. A., Souza, D. P., Ulson de Souza, A. A., and Guelli U. de Souza, S. M. A. "Prediction of Effective Diffusivity Tensors for Bulk Diffusion with Chemical Reactions," Brazilian Journal of Chemical Engineering, 24, 47-60, 2007.

6. Brenner, H. "Dispersion Resulting From Flow through Spatially Periodic Porous Media," Trans. Royal. Soc. 297.81-133, 1980.

7. Carbonell, R. G. and Whitaker, S. "Dispersion in Pulsed Systems-II Theoretical Developments for Passive Dispersion in Porous Media," Chem. Eng. Sci., 38, 1795-1802, 1983.

8. Carbonell, R. G.; Whitaker, S. Heat and Mass Transfer in Porous Media. In Fundamentals of Transport Phenomena in Porous Media; Bear, J., Corapcioplu, M. Y., Eds.; NATO Advanced Study Institute Series E 82; Marinus Nijhoff Publishers: Dordrecht, The Netherlands, p. 121. 1984.

9. Chang, H.-C. "Multiscale Analysis of Effective Transport in Periodic Heterogeneous Media," Chem. Eng. Commun., 15, 83-91, 1982.

10. Chang, H.-C. "Effective Diffusion and Conduction in Two-Phase Media: a Unified Approach,” A.1.Ch.E. J. 29, 846-853, 1983. 
11. Chu, K. T. and Bazant, M. Z. "Nonlinear Electrochemical Relaxation around Conductors," Phys. Rev. E 74, 011501, 2006.

12. Chu, K. T. and Bazant, M. Z. "Surface Conservation Laws at Microscopically Diffuse Interfaces." Journal of Colloid and Interface Science, 315, 319-329, 2007.

13. Corry, B., Kuyucak, S., and Chung, Sh.-H. "Dielectric Self-Energy in Poisson-Boltzmann and Poisson-Nernst-Planck Models of Ion Channels," Biophysical J., 84, 3594-3606, 2003.

14. Dukhin, S. S. "Electrochemical Characterization of the Surface of a Small Particle and Nonequilibrium Electric Surface Phenomena," Adv. Colloid Interface Sci., 61, 17-49, 1995.

15. Dukhin, S. S., Zimmermann, R., and Werner, C. "A Concept for the Generalization of the Standard Electrokinetic Model," Colloid Surf. A, 195, 103-112, 2001.

16. Gabitto, J. F. and Tsouris, C., "Simulation of the Capacity Deionization Process in Homogeneous Porous Electrodes," Transport in Porous Media, http://dx.doi.org/10.1007/s11242-015-0502-0, 0169-3913, 05/2015.

17. Gray, W. G. "A Derivation of the Equations for Multiphase Transport," Chem. Eng. Sci. 30, 229, 1975.

18. Gray, W. G. and Lee, P. C. Y. "On the Theorems for Local Volume Averaging of Multiphase Systems.” Int. J. Multiphase Flow, 3, 333-340, 1977.

19. Ho, F.-G. and Strider, W. "A Variational Calculation of the Effective Surface Diffusion Coefficient and Tortuosity," Chem. Eng. Sci., 36, 253-258, 1981.

20. Johnson, A. M. and Newman, J. "Desalting by Means of Carbon Electrodes," J. Electrochem. Soc., 118, 510-517, 1971.

21. Johnson, D. L. and Sen, P. N. "Dependence of the Conductivity of a Porous Medium on Electrolyte Conductivity,” Phys. Rev. B, 37, 3052, 1988.

22. Kim, J. H.; Ochoa, J. A.; and Whitaker, S. "Diffusion in Anisotropic Porous Media," Transport in Porous Media, 2, 327-356, 1987.

23. Kuwabara, S. "The Forces Experienced by a Lattice of Elliptic Cylinders in a Uniform Flow at Small Reynolds Numbers.” J. of Phys. Soc. Jpn., 14(4), 522-527, 1959.

24. Leroy, P. and Revil, A. "A Triple-Layer Model of the Surface Electrochemical Properties of Clay Minerals,” Journal of Colloid and Interface Science, 270, 371-380, 2004.

25. Looker, J. R. and Carnie, S; L. "Homogenization of the Ionic Transport Equations in Periodic Porous Media," Transport in Porous Media, 65, 107-131, 2006.

26. Lyklema, J., and Minor, M. "On Surface Conduction and its Role in Electrokinetics," Colloid Surf. A, 140, 33-41, 1998.

27. Maxwell, J. C. "Treatise on Electricity and Magnetism," Vol. I. Clarendon Press, Oxford, 1881. 
28. Mirzadeh, M., Gibou, F., and Squires, T. M. "Enhanced Charging Kinetics of Porous Electrodes: Surface Conduction as a Short-Circuit Mechanism.” Phys. Rev. Lett., 113, $097701,2014$.

29. Mohajeri, A., Narsilio, G. A., Peter Pivonka, P., and Smith, D. W. "Numerical Estimation of Effective Diffusion Coefficients for Charged Porous Materials Based on Micro-Scale Analyses," Computers and Geotechnics, 37, 280-287, 2010.

30. Newman, J. and Thomas-Alyea, K. E., Electrochemical Systems, 3rd ed. Wiley, New York, Chap. 22, 2004.

31. Niessner, J. and Helmig, R. "Multi-Scale Modeling of Three-Phase-Three-Component Processes in Heterogeneous Porous Media," Advances in Water Resources, 30, 2309-2325, 2007.

32. Nozad, I.; Carbonell, R. G.; Whitaker, S. "Heat Conduction in Multiphase Systems-I. Theory and Experiment for Two-Phase Systems," Chem. Eng. Sci., 40, 843, 1985.

33. Ochoa, J. A. "Diffusion and Reaction in Heterogeneous Media." Ph.D. Thesis, Department of Chemical Engineering, University of California at Davis, 1988.

34. Ochoa-Tapia, J. A.; Del Rio, J. A.; and Whitaker, S. "Bulk and Surface Diffusion in Porous Media: An Application of the Surface-Averaging Theorem," Chem. Eng. Sci., 48, 2061-2082, 1993.

35. Pivonka, P., Smith, D., and Gardiner, B. "Investigation of Donnan equilibrium in Charged Porous Materials - A Scale Transition Analysis," Transport in Porous Media, 69, 215-237, 2007.

36. Pivonka, P., Narsilio, G., Li R, Smith, D., and Gardiner, B. "Electrodiffusive Transport in Charged Porous Media: From the Particle-Level Scale to the Macroscopic Scale Using Volume Averaging," Journal of Porous Media. 12, 101-118, 2009.

37. Porada, S., Zhao, R., Van der Wal, A, Presser, V., and Biesheuvel, P. M. "Review on the Science and Technology of Water Desalination by Capacitive Deionization," Progress in Material Science, 58, 1388-1442, 2013.

38. Pride, S. R. "Governing Equations for the Coupled Electromagnetics and Acoustics of Porous Media,” Phys. Rev. B 50, 15678-15696, 1994.

39. Quintard, M. "Diffusion in Isotropic and Anisotropic Porous Systems: Three-Dimensional Calculations," Transport in Porous Media, 11, 187-199, 1993.

40. Rayleigh, R. S. "On the Influence of Obstacles Arranged in Rectangular Order upon the Properties of the Medium," Phil. Mag., 34, 481-489, 1892.

41. Revil, A. "Ionic Diffusivity, Electrical Conductivity, Membrane and Thermoelectric Potentials in Colloids and Granular Porous Media: A Unified Model," Journal of Colloid and Interface Science, 212, 503-522, 1999. 
42. Revil, A., Darot, M., and Pezard, P. "From Surface Electrical Properties to Spontaneous Potentials in Porous Media," Surveys in Geophysics, 17, 331-346, 1996.

43. Revil, A. and Glover, P. W. J. "Theory of Ionic-Surface Electrical Conduction in Porous Media," Physical Review B, 55, 1757, 1997.

44. Revil, A. and Glover, P. W. J. "Nature of Surface Electrical Conductivity in Natural Sands, Sandstones, and Clays," Geophysical Research Letters, 25, 691-694, 1998.

45. Revil, A., and Jougnot, D. "Diffusion of Ions in Unsaturated Porous Materials," Journal of Colloid and Interface Science, 319, 226-235, 2008.

46. Revil A., and Linde N. "Chemico-Electromechanical Coupling in Microporous Media," Journal of Colloid and Interface Science, 302, 682-94, 2006.

47. Revil, A., Pezard, P., and Darot, M.“ Electrical Conductivity, Spontaneous Potential and Ionic Diffusion in Porous Media," Geological Society London Special Publications, 122, 253-275, 1997.

48. Ryan, D., Carbonell, R. G. and Whitaker, S. "Effective Diffusivities for Catalyst Pellets under Reactive Conditions.” Chem. Eng. Sci., 35, 10-16, 1980.

49. Samson, E., Marchand, J., Snyder, K. A., and Beaudoin, J. J. "Modeling Ion and Fluid Transport in Unsaturated Cement Systems in Isothermal Conditions," Cement and Concrete Research, 35, 141- 153, 2005.

50. Schwartz, L. M., Sen, P. N., and Johnson, D. L. "Influence of Rough Surfaces on Electrolytic Conduction in Porous Media," Physical Review B, 40, 2450-2458, 1989.

51. Sharma, K., Mayes, R. T., Kiggans jr., J. O., Yiacoumi, S., Gabitto, J., DePaoli, D. W., Dai, S., and Tsouris, C. "Influence of Temperature on the Electrosorption of Ions from Aqueous Solutions using Mesoporous Carbon Materials." Separation and Purification Technology, 116, 206-213, 2013.

52. Sharma, K., Kim, Y,-H., Gabitto, J., Mayes, R. T., Yiacoumi, S., Bilheux, H. Z., Walker, L. M.H., Dai, S. and Tsouris, C. "Transport of Ions in Mesoporous Carbon Electrodes during Capacitive Deionization of High-Salinity Solutions," Langmuir, 31, 1038-1047 (2015).

53. Scheiner, S., Pivonka, P., and Smith, D. "Two-Scale Model for Electro-Diffusive Transport through Charged Porous Materials.” IOP Conf. Series: Materials Science and Engineering, 10, 012112, 2010.

54. Schmuck, M. "Modeling and Deriving Porous Media Stokes-Poisson-Nernst-Planck Equations by a Multiple-Scale Approach," Communications in Mathematical Sciences, 9, 685-710, 2011.

55. Schmuck, M. and Bazant, M. Z. "Homogenization of the Poisson-Nernst-Planck Equations for Ion Transport in Charged Porous Media," arXiv:1202.1916v2, 2014.

56. Tiedemann, W., and Newman, J. "Porous-Electrode Theory with Battery Applications," AIChE J., 21, 25, 1975. 
57. Ulson de Souza, A. A., and Whitaker, S. "The Modeling of a Textile Dyeing Process Utilizing the Method of Volume Averaging," Brazilian Journal of Chemical Engineering, 20, 445-453, 2003.

58. Valdes-Parada, F. J. and Alvarez-Ramirez, J. "On the Effective Diffusivity under Chemical Reaction in Porous Media," Chem. Eng. Sci., 65, 4100-4104, 2010.

59. Volfkovich, Y. M., Mikhalin, A. A., and Rychagov, A. Y. "Surface Conductivity Measurements for Porous Carbon Electrodes." Russian Journal of Electrochemistry, 49, 594598, 2013.

60. Whitaker, S. "Local Thermal Equilibrium: An Application to Packed Bed Catalytic Reactor Design," Chem. Eng. Sci., 41, 2029, 1986.

61. Whitaker, S., "The Method of Volume Averaging." Kluwer Academic Publishers, Netherlands, 1999.

62. Zhao, R., Biesheuvel, P. M., Miederna, H., Bruning, H., and van der Wal, A. "Charge Efficiency: A Functional Tool to Probe the Double-Layer Structure inside of Porous Electrodes and Application in the Modeling of Capacitive Deionization," J. of Phys. Chem. Lett., 1, 205-210, 2010.

63. Zukoski IV, C. F. and Saville, D. A. "An Experimental Test of Electrokinetic Theory using Measurements of Electrophoretic Nobility and Electrical Conductivity," J. of Colloid and Interface Sci., 107, 322-333, 1985.

64. Zukoski IV, C. F. and Saville, D. A. "The Interpretation of Electrokinetic Measurements using a Dynamic Model of the Stern Layer: I. The Dynamic Model," J. of Colloid and Interface Sci., 114, 32-44, 1986. 


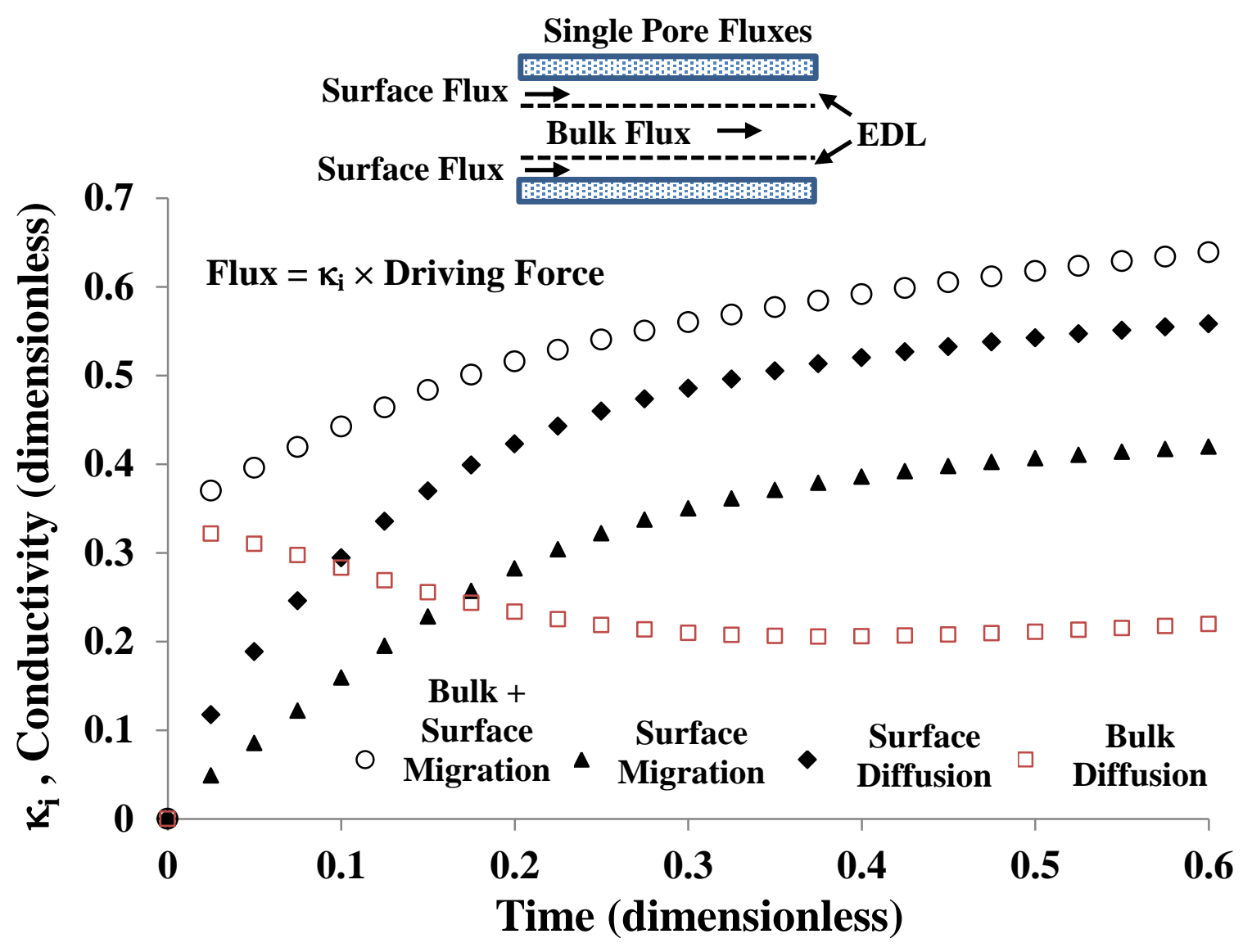

Different dimensionless contributions to electrolyte conductivity in porous media. 